\title{
Implementation of standard testbeds for numerical relativity
}

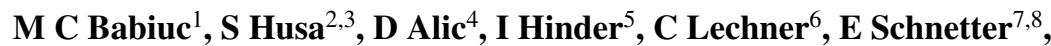 \\ B Szilágyi $^{3}$, Y Zlochower ${ }^{9}$, N Dorband ${ }^{3}$, D Pollney ${ }^{3}$ and J Winicour ${ }^{3,10}$ \\ ${ }^{1}$ Department of Physics and Physical Science, Marshall University, Huntington, WV 25755, USA \\ 2 Friedrich Schiller University Jena, Max-Wien-Platz 1, 07743 Jena, Germany \\ ${ }^{3}$ Max-Planck-Institut für Gravitationsphysik (Albert-Einstein-Institut), Am Mühlenberg 1, \\ 14076 Golm, Germany \\ ${ }^{4}$ Department of Physics, University of the Balearic Islands, Cra Valldemossa km 7.5, \\ 07122 Palma de Mallorca, Spain \\ ${ }^{5}$ Center for Gravitational Wave Physics, The Pennsylvania State University, University Park, \\ PA 16802, USA \\ ${ }^{6}$ Weierstrass Institute for Applied Analysis and Stochastics (WIAS), Mohrenstraße 39, \\ 10117 Berlin, Germany \\ ${ }^{7}$ Center for Computation \& Technology, 216 Johnston Hall, Louisiana State University, \\ Baton Rouge, LA 70803, USA \\ ${ }^{8}$ Department of Physics and Astronomy, 202 Nicholson Hall, Louisiana State University, \\ Baton Rouge, LA 70803, USA \\ ${ }^{9}$ Center for Computational Relativity and Gravitation, School of Mathematical Sciences, \\ Rochester Institute of Technology, 78 Lomb Memorial Drive, Rochester, New York 14623, USA \\ ${ }^{10}$ Department of Physics and Astronomy, University of Pittsburgh, Pittsburgh, \\ Pennsylvania 15260, USA
}

Received 17 September 2007, in final form 21 April 2008

Published 2 June 2008

Online at stacks.iop.org/CQG/25/125012

\begin{abstract}
We discuss results that have been obtained from the implementation of the initial round of testbeds for numerical relativity which was proposed in the first paper of the Apples with Apples Alliance. We present benchmark results for various codes which provide templates for analyzing the testbeds and to draw conclusions about various features of the codes. This allows us to sharpen the initial test specifications, design a new test and add theoretical insight.
\end{abstract}

PACS numbers: $\quad$ 44.70.Bw, 04.25.Dm, 04.40.Nr, 98.80.Cq

\section{Introduction}

For decades, the field of numerical relativity has been dominated by an often painful quest for stable black-hole inspiral simulations. More than 40 years after Hahn and Lindquist's first pioneering numerical simulation of colliding black holes [1], this quest has recently turned into a gold rush when Pretorius's breakthrough simulation [2] based on a harmonic code was 
followed by simultaneous invention of the 'moving punctures' method by two independent groups $[3,4]$.

The primary motivation for solving the binary black-hole problem in numerical relativity has however been to supply waveforms for gravitational wave detectors. This goal demands an approach that goes beyond the efforts that have lead to an explosion in publications from the binary black-hole community. Cross-validation of waveforms between different groups (and codes) and comparison with post-Newtonian predictions will be essential for numerical waveforms to be used in the computationally expensive searches conducted by the international gravitational wave community. The importance of cross-validation of numerical relativity results as a community effort was foreseen by the Apples with Apples Alliance (AwA) [5], which has presented a first round of standardized testbeds [6]. This first round comprises four tests with periodic boundaries, designed to efficiently exhibit code instability and inaccuracy. Instabilities currently receive less attention, since it has turned out that, paradoxically, binary black-hole evolutions are in some sense a simpler problem than had been expected, and current codes evolving binary black holes do not typically show instabilities. The same codes will however have difficulties with some of the testbeds presented in the first round. The theoretical understanding of what works and what does not in numerical relativity is still very much an open problem. One crucial theoretical advance, which has been made since the publication of our first paper [6], is the development of a theory for well-posed second order in space, first order in time systems [7-13], which has been extended to a basic understanding of numerical stability for such systems [11-13].

Over the past years several groups have committed their test results to a publicly available data repository, with activities being coordinated via the website http://www. ApplesWithApples.org. The purpose of the present paper is to document these developments and discuss their feedback with respect to code performance, to test improvement and to design further tests. While predating the binary black-hole breakthroughs, we believe that the initial Apples with Apples tests and results are still valuable as providing a first testbed for a community effort in numerical relativity.

The tests side-step many issues that would arise in a precise discussion of the binary black-hole problem, such as the issue of boundaries. We make the natural choice of periodic boundaries for a first round of tests to isolate the performance of evolution algorithms. This is equivalent to evolution on the topology of a 3-torus in the absence of boundaries. However, in the context of general relativity, this introduces complications of a cosmological nature regarding the instability of Minkowski spacetime to perturbations on a compact manifold, as has been discussed in [6].

Establishing a paradigm for standardized testbeds for numerical relativity is a formidable task in itself. We can draw on experience from other fields, such as computational hydrodynamics where such testbeds have been used for a long time (for an overview of CFD testbed resources on the web, see, e.g., [14]; for an example of initial-value ordinary differential equation (ODE) test-suites see [15]). However, general relativity comes with its own issues that introduce extra complications. First of all, it is important to realize that the numerical relativity community is small, with very limited available manpower. In contrast to the size of the field, we are trying to solve many difficult problems at the same time. Numerical methods are being developed in parallel with the formulation of the continuum problem, with the construction of physically relevant initial data sets and with the unraveling of the physical processes involved in the systems under investigation. All of this is, so far, without the help of comparison with experiments. Groups working in the field are faced with many fundamental questions in designing their approaches. Codes are in a state of flux that makes careful documentation easy to postpone. A good example is the issue of boundaries, which can be 
taken to be either a cubic grid boundary or a smooth spherical boundary, which can either be mapped to infinity or given some finite artificial location, and which are further complicated by gauge freedom and the requirements of constraint preservation. Useful comparison of the wide variety of resulting codes requires simple tests which isolate an important facet of the problem.

We distinguish two fundamentally different types of testbed: the first type compares different codes and methods in the treatment of a physically interesting set of solutions. In the context of the binary black-hole problem, a detailed comparison of nonspinning equal-mass inspiral would be a natural example. The second type is idealized situations, such as the 'shock tube test' [16] in computational fluid dynamics. This is the type of testbed we discuss in the present paper, where we restrict ourselves to a greatly simplified first set of tests [6]: periodic grids and strict test specifications, which as far as practicable define all the details of a simulation except the formulation of the Einstein equations. Our experience with the first round of testbeds confirms this decision: even the analysis of these simple situations has proved quite challenging. Our conclusions in section 8 discuss how the experience from the present round of tests can be used in our development of black-hole tests.

We identify five main aims of standardized tests of the 'idealized' type:

(i) Standardized tests should provide the young and fast-changing community of numerical relativists with a common reference frame which will help integrate different efforts to produce a coherent picture of what works and what does not, and thus reduce the dependence on anecdote and fashion.

(ii) Tests should be efficient in revealing instabilities or other weaknesses of an algorithm, both regarding simplicity of the analysis, run time and implementation.

(iii) Tests should help identify where problems come from, as a step toward improvement of the algorithms.

(iv) Tests should facilitate comparisons between approaches regarding different continuum formulations, spatial discretizations, time integrators, uses of artificial dissipation, etc.

(v) The development of testbeds should eventually lead to useful code comparisons for judging the validity of physically interesting simulations, e.g. the binary black-hole problem.

Point (i) has been addressed by organizing this project as a community initiative, which seeks broad participation and provides test results via web pages and a CVS repository [5]. Regarding point (ii), in this paper we review our original test specifications and propose modifications to promote efficiency. Point (iii) is essential for the character of this paper: we focus on presenting test results as a template for analyzing and interpreting results, rather than just presenting the broadest possible listing of test output for a maximal number of codes. We feel that it is essential to stress this point: tests which do not directly correspond to a physically interesting situation are only valuable if they improve our understanding of what really goes on with a certain code. Only then can we hope to carry over test benefits to other situations. Such analysis does of course require a certain effort.

Point (iv) is dealt with by providing 'standard candle results' in the CVS repository, i.e., benchmarks that have been obtained with very strictly defined specifications. Point (v) represents the ultimate goal of the AwA Alliance.

The analysis of test results has led to better understanding of the four original standardized tests and has led to some improvements in their specifications. We also have added a new shifted gauge wave test, which closes a gap regarding the ability of a code to handle a shift. The revised specifications for the five tests are detailed in appendix A. The major changes from the specifications in [6] are 
- Robust stability test. The rules for how the data should scale with resolution have been changed; the criteria for passing the test have been restated.

- Linearized wave test. No changes.

- Gauge wave test. The original tests amplitudes $A=0.01$ and $A=0.1$ have been replaced with $A=0.5$.

- Shifted gauge wave test. This new test has been added.

- Gowdy wave test. No changes.

We have also dropped the original requirement that the tests be run with a iterative CrankNicholson integrator. Conclusions from the test results and our experiences with the testing procedures, along with the reasons behind the changes and additions in the standard tests, are summarized in section 8 .

The code descriptions and test data on which this paper is based are described in section 2. The results for the original four standardized tests are discussed in sections 3-5 and 7. Discussion of the shifted gauge wave test and some benchmarks are given in section 6 .

The plots presented in this paper are based upon test output in the CVS repository. Many of these tests were run with codes in which artificial dissipation was only introduced implicitly through the use an iterated Crank-Nicholson (ICN) time integrator. It had been a naive hope at the beginning of this project that the use of ICN might provide a way to standardize the introduction of dissipation. Most numerical relativity groups now use Runge-Kutta time integrators with the explicit addition of Kreiss-Oliger dissipation (see appendix C.2). It has been found that many of the test results presented here could be greatly improved by such explicit use of dissipation. In addition to artificial dissipation, most codes that simulate binary black holes use higher order approximations than the second-order accurate codes being compared here. Consequently, we want to emphasize that the results exhibited in this paper should not be used to make judgments on particular approaches, but that our purpose is to assess and improve the test suite and to provide a basis for future code comparisons.

\section{Code descriptions}

In order to ensure a consistent presentation of test output, we present a brief account of the numerical codes and algorithms which have been used to produce the data on which this paper is based. All data are publicly available via the CVS repository (see [5] for details). The four original standardized tests are denoted by ROBUST (the robust stability test), LINEAR (the linear wave test), GAUGE (the gauge wave test) and GOWDY (the Gowdy wave test). Table 1 summarizes the output data that have been submitted for the various codes.

The usefulness of these data depends upon good code documentation. It is beyond the scope of this paper to provide such documentation for all the codes involved. However, we will outline some basic code information which is necessary to interpret the test results. The complexity of this task is somewhat alleviated because all the codes represented here follow a method of lines approach. We will organize the code descriptions along the following guidelines.

- A description of the continuum formulation, including a list of all variables, their associated evolution equations and constraints (both differential and algebraic), equations governing the lapse and shift and a specification of any free parameters. Terms and differential operators in the equations should be ordered in the way that they are approximated by finite difference expressions in order to avoid ambiguities associated with the Leibniz rule. The hyperbolicity classification should be provided, if known. 
Table 1. Test output and codes considered in this paper. The code abbreviations are explained below, along with a description of the finite difference algorithm. A '++' indicates a full complement of test output in the CVS, a ' + ' indicates partial output which has been used for our analysis, a '-' indicates partial output on which no meaningful conclusions could be drawn and a ' -- ' indicates no output.

\begin{tabular}{lllll}
\hline CODE & ROBUST & LINEAR & GAUGE & GOWDY \\
\hline Abigel_harm & ++ & ++ & ++ & ++ \\
AEI_CactusEinsteinADM & + & -- & -- & ++ \\
Kranc_FreeADM & + & + & + & + \\
CCATIE_BSSN & ++ & ++ & ++ & ++ \\
Kranc_BSSN & ++ & ++ & ++ & ++ \\
LazEv_BSSN & ++ & ++ & ++ & ++ \\
HarmNaive & ++ & ++ & ++ & ++ \\
KrancNOR & ++ & ++ & ++ & ++ \\
KrancFN & ++ & ++ & ++ & -- \\
LSU_HyperGR & ++ & ++ & ++ & ++ \\
\hline
\end{tabular}

- A description of the semi-discrete system, describing the spatial finite difference equations on each time level, including the rules for discretizing partial derivatives as centered or onesided finite differences and any other discretization techniques, such as spatial averaging or dissipation. For complicated systems, the finite difference rules may be specified only for the principal part, with further details supplied by references. (Here we provide some basic reference material in appendices $\mathrm{B}$ and $\mathrm{C}$ for compactness of presentation.)

- A description of the numerical time update scheme. All manipulations of data between intermediate time steps should be specified, such as enforcing a constraint.

As an example, we consider two inequivalent algorithms for the wave equation $\square \phi=0$ (with unit lapse, zero shift and spatial metric $\gamma_{i j}$ ), which should be expected to result in different code performance. In both cases the second order in time system is reduced to first order in time by introducing the variable $\pi=\partial_{t} \phi$, and applying, say, fourth-order RungeKutta (see appendix C) to the ODEs of the semi-discrete system obtained using the method of lines. Two different codes can based upon the following descriptions.

Description I:

(i) The continuum system is

$$
\begin{aligned}
\partial_{t} \phi & =\pi, \\
\partial_{t} \pi & =\frac{1}{\sqrt{\gamma}} \partial_{i}\left(\sqrt{\gamma} \gamma^{i j} \partial_{j} \phi\right) .
\end{aligned}
$$

(ii) The semi-discrete version is obtained by replacing all partial derivatives in (2) by centered differences:

$$
\partial_{t} \pi=\frac{1}{\sqrt{\gamma}} D_{0 i}\left(\sqrt{\gamma} \gamma^{i j} D_{0 j} \phi\right),
$$

where $D_{0 i}$ is the centered difference operator $D_{0}$ applied in direction $i$ (see appendix C.1).

Description II (inequivalent with I):

(i) The continuum system is

$$
\partial_{t} \phi=\pi
$$




$$
\partial_{t} \pi=\gamma^{i j} \partial_{i} \partial_{j} \phi+\frac{1}{\sqrt{\gamma}} \partial_{i}\left(\sqrt{\gamma} \gamma^{i j}\right) \partial_{j} \phi .
$$

(ii) The semi-discrete version is obtained by replacing the partial derivatives in (2) by centered differences according to

$$
\gamma^{i j} \partial_{i} \partial_{j} \phi+\frac{1}{\sqrt{\gamma}} \partial_{i}\left(\sqrt{\gamma} \gamma^{i j}\right) \partial_{j} \phi=\gamma^{i j} D_{+i} D_{-j} \phi+\frac{1}{\sqrt{\gamma}} D_{0 i}\left(\sqrt{\gamma} \gamma^{i j}\right) D_{0 j} \phi,
$$

where $D_{+i}$ and $D_{-i}$ represents forward and backward centered finite differences in the respective directions (see appendix C.1).

The codes resulting from these two descriptions produce substantially different performance because of the 'checkerboard' design of the stencil used in description $\mathbf{I}$. Descriptions of the specific codes used in this paper are given in appendix B.

\section{Robust stability test}

The robust stability test was intended as a first screen to eliminate many unstable evolution algorithms. The particular importance of this test was due to the fact that instabilities of numerical codes appeared as a prime obstacle to 'solve' the binary black-hole problem, and essentially no theoretical understanding was available to discuss the well-posedness and numerical stability of first order in time, second order in space formulations of the Einstein equations, which have been and still are popular in the field. Recently, a theoretical framework has become available to discuss the well-posedness and numerical stability of such mixed order formulations of the Einstein equations [7-13, 17, 18], and it has been extended to the problem of discretizing the equations in the context of the method of lines [11-13]. As a consequence of both the recent breakthroughs in the binary black-hole problem and the theoretical advances, numerical stability has become a relatively minor issue in practice (although there certainly remain interesting mathematical questions to be pursued). We thus restrict ourselves to a minimal discussion here, as is sufficient to understand the data available in our test results repository. For a more in-depth discussion of theoretical and practical aspects of numerical stability and the robust stability test we refer to [11], which has been directly motivated by numerical results obtained within this project.

While the other tests give quantitative information about an evolution system, e.g. the magnitude of the numerical error, the result of the robust stability test is 'pass' or 'fail'. A stable numerical algorithm is only possible if the underlying continuum problem is well-posed [19]. In the well-posed case an instability might still arise, either from the numerical technique or from the existence of an exponential mode in the continuum problem. The test is designed to avoid continuum instabilities by considering small perturbations of the Minkowski metric. In addition to providing efficient detection of unstable numerical algorithms (or coding errors) affecting the principal part of the evolution system, it is also intended to spot instabilities arising from ill-posed systems, such as weakly hyperbolic systems.

As an example, consider the weakly hyperbolic system

$$
\begin{aligned}
& u_{, t}=u_{, x}+v_{, x} \\
& v_{, t}=v_{, x}
\end{aligned}
$$

with the periodic solutions

$$
\begin{aligned}
& u=\omega t \cos \omega(t+x), \quad v=\sin \omega(t+x) \\
& \omega=2 \pi m, \quad m=1,2,3, \ldots
\end{aligned}
$$


on the domain $-0.5 \leqslant x \leqslant 0.5$. In terms of the $L_{2}$-norm

$$
N=\left(\int_{-0.5}^{0.5}\left(u^{2}+v^{2}\right) \mathrm{d} x\right)^{1 / 2},
$$

the Cauchy data for (7) at $t=0$,

$$
u=0, \quad v=\sin \omega x,
$$

have norm $N(0)=1 / \sqrt{2}$. However, because of $(7), N(t) \sim \omega t$ for large $\omega$. This leads to a violation of the well-posedness requirement that in any finite time interval

$$
N(t)<A \mathrm{e}^{K t} N(0)
$$

in terms of constants $A$ and $K$ independent of the Cauchy data.

For discretized systems we cannot test well-posedness directly, but rather we test the analogous concept of numerical stability, i.e., we aim at establishing the existence of constants $A$ and $K$, which give rise to the bound

$$
\frac{\left\|v^{n}\right\|}{\left\|v^{0}\right\|} \leqslant A \mathrm{e}^{K t_{n}}
$$

where $v^{n}$ is the solution of the discrete system at time $t_{n}=n k$. The test is passed if such a bound can be established, and is failed otherwise. In the discretized version of a weakly hyperbolic problem, with grid displacement $h$, the perturbation of a simulation by random initial data can be expected to excite numerical error which grows linearly in time according to $u \sim t / h$, corresponding to the shortest wave number $\omega \sim 1 / h$. This would then lead to secular error growth which increases with resolution. Although the system (6) is well-posed with respect to a stronger norm including a $v_{, x}^{2}$ term, a generic perturbation of (6) by lower order terms would nevertheless produce an exponentially growing instability which cannot be bounded. See [20] for a more general discussion of such weakly hyperbolic systems.

The key idea of setting initial data for this test is to distribute energy roughly equally over all frequencies. This is a particularly efficient way to reveal growing modes if the growth rate increases with resolution, as is the case if the discretization is unstable or if the continuum problem is ill-posed. In our test we use a spectrum generated by random initial data.

The robust stability test as formulated here tests numerical stability in the linear, constant coefficient regime. It is based upon small random perturbations of Minkowski space, with the initial data consisting of random numbers $\epsilon$ applied at each grid point to every code variable requiring initialization. In numerical evolution, where machine precision takes the place of $\epsilon$, a code that cannot stably evolve such random noise would be unable to evolve smooth initial data.

In spite of its simplicity, our experience has shown that the robust stability test exhibits various subtle difficulties in designing a single test prescription that is universally effective for all evolution systems and numerical methods. Some particular problems are:

- For random initial data, where a significant part of the total energy is in high frequencies, dissipation has a large effect. Some intrinsic dissipation is unavoidable in finite difference evolution algorithms, and adding artificial dissipation may be necessary to stabilize certain algorithms [11], and insufficient to stabilize others (such as algorithms for weakly hyperbolic systems). Simulations of variable coefficient, nonlinear systems normally require numerical dissipation to obtain a stable evolution, e.g. by adding Kreiss-Oligertype dissipation [20] (see appendix C.2). Dissipation can however increase the time scale on which instabilities become apparent. The detailed way dissipation affects instabilities varies with the spatial discretization (we only consider second-order approximations here), with the time integrator, with the grid resolution and with the Courant number. 
- As discussed in the above example, well-posedness and numerical stability are defined with respect to a certain norm. Using an inappropriate norm can yield misleading results. Second-order systems require different norms than first-order systems [11].

- Numerical stability of an explicit time integration algorithm can only be expected if the time step is appropriately restricted by a Courant-Friedrichs-Lewy (CFL) condition. It is important to distinguish between resolution-dependent blowup associated with illposedness from blowup resulting from a CFL violation. For sufficiently complicated 3D algorithms, the CFL limit might not be readily deduced from analytic arguments. As an example, exponential growth of the ADM algorithm was mistakenly provided as an illustration of a failed robust stability test in [6]. It took subsequent testing and analysis to reveal that this exponential growth resulted from a CFL violation and that otherwise the weakly hyperbolic instability of ADM resulted in a secular (linear in time) growth.

As a result of such considerations, we will not try to present a single universally applicable specification for the robust stability test. Instead, while keeping the original spirit of the test as a simple and useful first screen, we propose some changes in the guidelines, as discussed below.

An important issue when performing stability tests is whether the high-frequency modes are damped. This has important bearing on the long-time behavior of the robust stability test: all damped modes will decay in time; eventually the undamped frequencies of the discrete system will dominate the signal. If an analysis of damping factors has not been performed, the test can therefore also be useful in detecting the spectrum of frequencies which are not damped. It has been pointed out in [11] for standard discretizations of first order in space systems that the 'checkerboard' mode is undamped, while for typical second-order systems it is damped. Since the 'checkerboard' mode is not realized on grids with an odd number of points, we adopt the practice of always using an even number of grid points so as not to muzzle such a potential instability.

In our original specifications, we proposed the relatively large time step $\mathrm{d} t=0.5 \mathrm{~d} x$, which turned out to be larger than the CFL limit for the ADM system. Since a smaller $\mathrm{d} t$ also decreases the amount of dissipation inherent in a time integrator, we now propose a relatively small time step to avoid distortion of results due to dissipation. Common time integrators in current practice in numerical relativity are ICN, RK3 and RK4 (sorted by decreasing internal amount of dissipation). A sufficiently small time step would yield similar results for all of them. We therefore propose to run with $\mathrm{d} t=0.1 \mathrm{~d} x$, which can be further reduced in case of doubt. See appendix A.1 for details.

For systems that use variables which correspond to spatial derivatives of the ADM 3metric and extrinsic curvature, an ambiguity arises: noise can be added uniformly to all variables, or to the ADM initial data before taking derivatives. There are similar ambiguities in second-order systems regarding how the range of the random numbers should scale with resolution. For uniformity of description, we propose to do the simplest thing, namely to apply noise to all evolution variables in the same way. We propose the range of $\pm 10^{-10}$ for all variables, the same range used for the lowest resolution in the original specifications.

Following common practice at the time, the Hamiltonian constraint was used to analyze test results. Again following [11], we now propose a pass/fail analysis based upon whether the time behavior of the norm satisfies (11).

Our core test specification combines both $1 \mathrm{D}$ and 3D features by running in a thin channel along the $x$-axis. The use of four distinct gridpoints in the $y$ - and $z$-directions allows for the checkerboard mode (ghost points may be necessary depending upon the numerical scheme). The generalization to a full cube 3D test is straightforward, and may add further clarification in case of dubious results. 

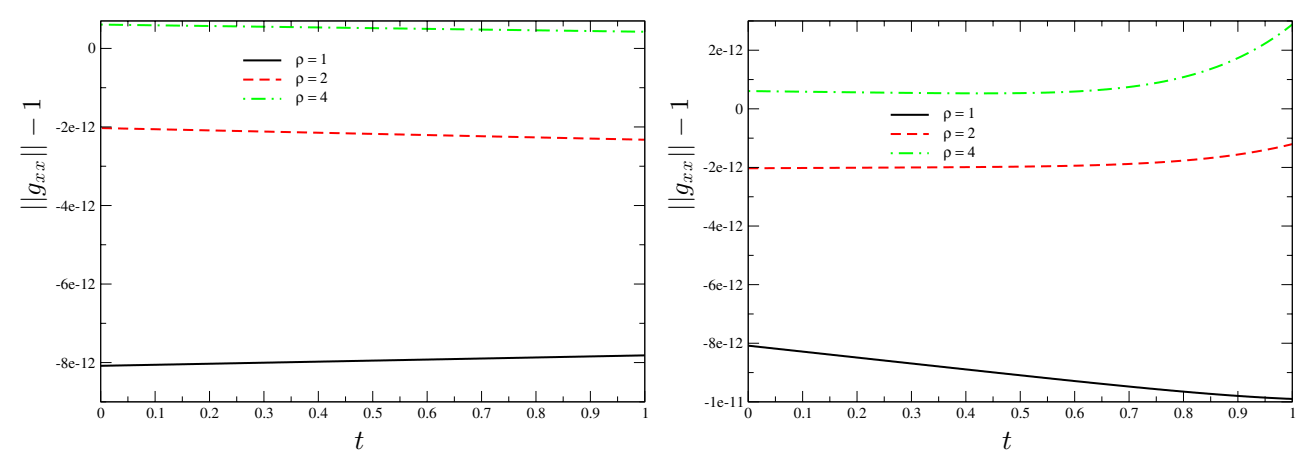

Figure 1. Convergence results for the robust stability test with the Abigel_harm (left) and HarmNaive (right) codes, for runs of one crossing time. The graphs show the error in $g_{x x}$ as a function of time, obtained by subtracting 1 from its $L_{2}$-norm. As seen from the slopes of the graphs, the Abigel_harm code (left) passes the test, because there is no increasing rate of error growth with higher resolution $\rho$, while the HarmNaive code (right) fails the test because the growth rate increases with resolution.

The test should be run until one is confident that dissipation effects do not cloud the result. Without artificial dissipation, a runtime of one crossing time, using output at every time step, is usually sufficient. This corresponds to $500 \rho$ time steps, for a given resolution $\rho$ (see appendix A). The test is passed if the norm satisfies the inequality (11) for all resolutions, for a fixed choice of $A$ and $K$.

Instabilities caused by the ill-posedness of the evolution system (or by coding errors in treating the principal part) are already apparent in one-dimensional tests, which can be performed quickly and economically. An example of how this analysis works is given in figure 1. The way that the slope of the error versus time depends upon resolution shows that the Abigel_harm code, which is based upon a symmetric hyperbolic formulation, passes the test; whereas the HarmNaive code, which is based upon a weakly hyperbolic formulation, fails the test.

\section{Linearized wave test}

A prime physical objective of numerical relativity is to compute the waveform from a system of black holes and neutron stars. This test checks the ability of a code to propagate a linearized gravitational wave, which is a minimally necessary attribute for reliable wave extraction from strong sources. Test specifications are given in appendix A.2.

The test checks the accuracy of the code in propagating both the amplitude and phase of the wave. It can reveal whether excessive dissipation has been necessary for good long-term performance in the robust stability test. For the $\rho=1$ coarsest grid ( $N=50$ grid zones), there is no enough resolution for second-order accurate codes to obtain accurate phase propagation and the corresponding runs should only be viewed as an economical first check on the code. The most useful comparisons are with the $\rho=4$ grid.

Figure 2 compares snapshots of the 1D wave after 1000 crossing times which were obtained with a variety of codes using the $\rho=4$ finest grid. For reference, the exact waveform is also plotted. The snapshots for three of the codes, Abigel_harm, HarmNaive and LazEv_BSSN, are very similar and provide a good benchmark for the accuracy that can be achieved at this resolution. They very closely match the exact solution in amplitude but 


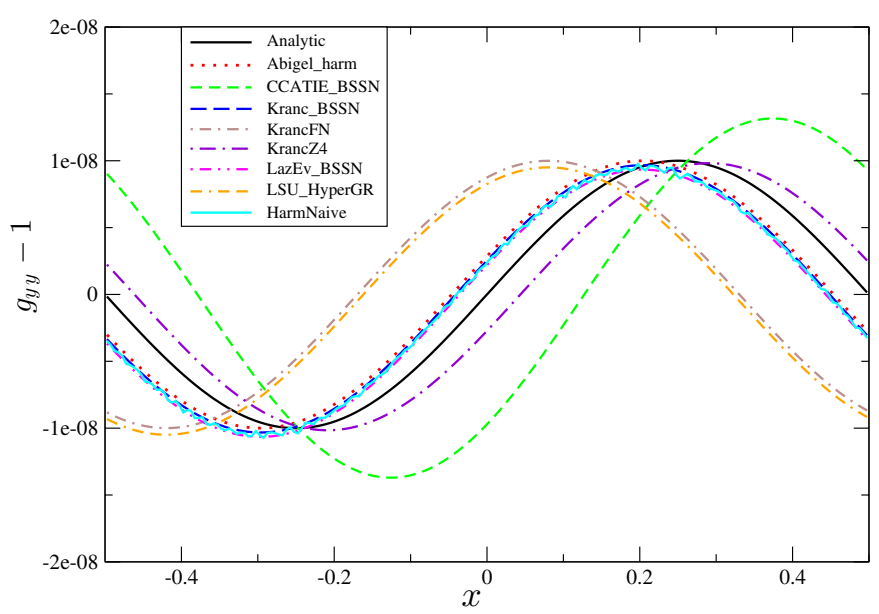

Figure 2. Comparison snapshots of $g_{y y}(x)-1$ at $t=1000$ for the $1 \mathrm{D}$ linearized wave test, with $\rho=4$ resolution.

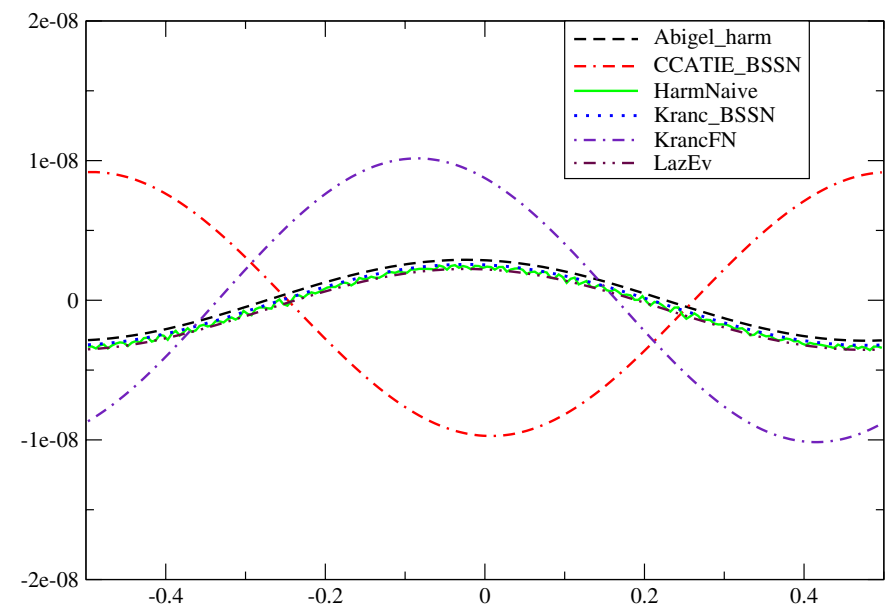

Figure 3. Comparison snapshots of the error $\mathcal{E}$ in $g_{y y}(x)$ at $t=1000$ for the $1 \mathrm{D}$ linearized wave test, with $\rho=4$ resolution.

show a phase delay, similar to the delay seen in the following gauge wave test. It should be expected that phase accuracy could be improved by going to fourth-order accurate methods. Some snapshots of the corresponding error are displayed in figure 3. Except for the two codes with the largest phase error, the error at 1000 crossing times is confined to a small band. By monitoring the growth of the error during the evolution, it was verified that no overall multiple of $2 \pi$ phase error is concealed in the snapshots of figure 2 .

In addition, the plots of the Hamiltonian in figure 4 show no rapidly growing constraint violating instabilities in this linear regime. The secular instability of Harm_Naive, which was discussed in the robust stability test, is evident but it does not introduce a large error in this test. This illustrates that instabilities associated with a weakly hyperbolic system are not 


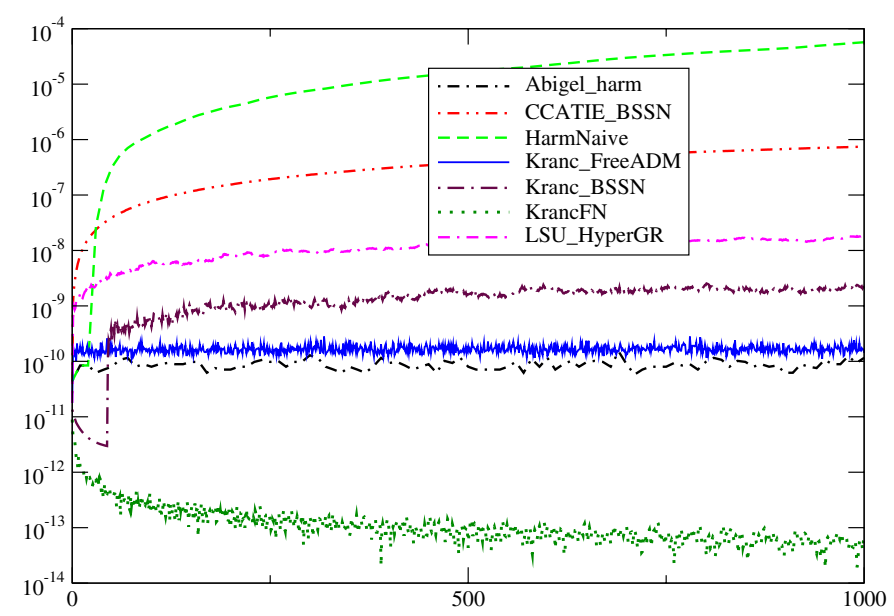

Figure 4. Comparison of time dependence of the $L_{\infty}$-norm of the Hamiltonian constraint $\|\mathcal{H}\|$, shown on a logarithmic scale, for the $1 \mathrm{D}$ linearized wave test with $\rho=4$ resolution.

necessarily evident in linearized tests where, as discussed in section 3 , the unstable modes only grow secularly in time. The KrancFN code gives good accuracy for the amplitude but a much larger error in phase. The CCATIE code shows poor accuracy in both phase and error. It is beyond the scope of this paper to explain the discrepancy between the performance of the two BSSN codes.

The 1D linear wave test is simple and economical to perform. Although the test is not very demanding, the results for the metric component $g_{y y}$ in figures 2 and 3 show that it provides a benchmark which can be useful to identify weaknesses in code performance. The 2D tests require more computer time and the results were typically in line with expectations from the $1 \mathrm{D}$ results.

\section{Gauge wave test}

The gauge wave test is based on a nonlinear gauge transformation of Minkowski spacetime. Although the correct solution is a flat spacetime, nonlinear effects and the nontrivial geometry of the time slices can easily trigger continuum instabilities in the equations. For simple examples of such effects see [21] for a nonlinear wave equation on flat space, designed to model problems arising in this testbed, and [22] for a linear example of how nontrivial geometry of the slicing can trigger instabilities already for the Maxwell equations.

Our original specifications [6] were to run the test with amplitudes $A=0.01$ and $A=0.1$. Many codes have been sufficiently improved to handle larger amplitudes, which is generally more efficient in detecting instabilities with smaller run times. Accordingly, we specify an amplitude of $A=0.5$ in the revised test details given in appendix A.3.

While the gauge wave metric has a rather simple form, the test proved to be challenging for most evolution codes. One anticipated source of growing error is the instability of a flat space with $T^{3}$ topology [6]. Another problem is the existence of a family of harmonic, exponential gauge modes corresponding to the substitution $H \rightarrow \mathrm{e}^{\lambda t} H$ (for arbitrary $\lambda$ ) into the metric (A.10) [21]. The testbed itself corresponds to $\lambda=0$, but numerical error can easily excite this mode and lead to exponential growth of the wave amplitude. Other instabilities may be present 


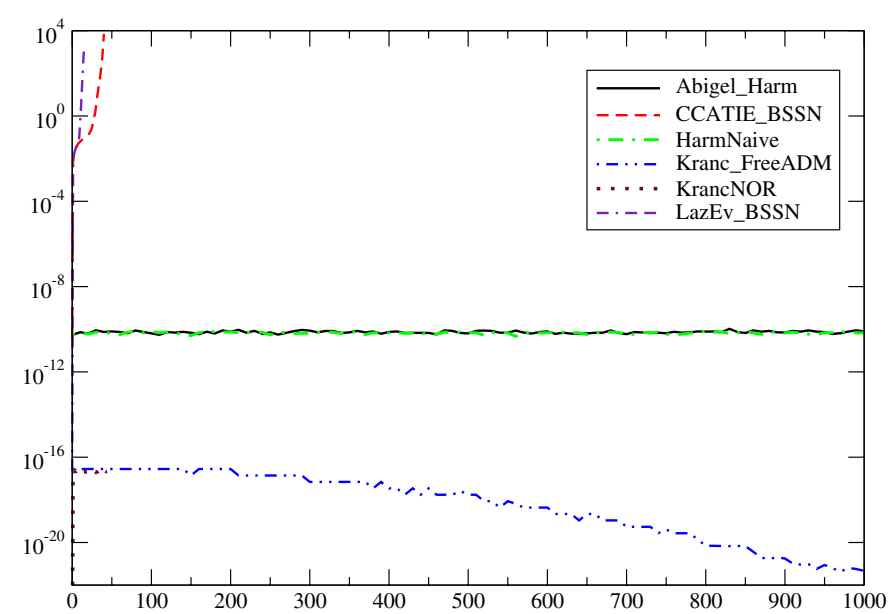

Figure 5. Time dependence of the $L_{\infty}$-norm of the Hamiltonian constraint $\|\mathcal{H}\|$, shown on a logarithmic scale, for the $1 \mathrm{D}$ gauge wave test with resolution $\rho=4$ and amplitude $A=0.1$.

in individual systems, depending on the detailed form of the reduced evolution system for the particular formulation. Some of these instabilities can be identified by looking at the growth of the constraints for the formulation. In addition to instabilities that correspond to solutions of the continuum problem, individual codes may suffer from numerical instabilities depending on the discretization schemes. These would typically be seen as high-frequency modes and, for well-posed systems, can be cured by adding artificial dissipation to the numerical algorithm.

Figure 5 shows the time evolution of the Hamiltonian constraint for the various codes. The negligible violation of the Hamiltonian constraint by the harmonic codes can be attributed to the fact that the harmonic coordinate conditions are used to shift the role of the constraint to an evolution equation. Note that the BSSN codes show rapid growth of Hamiltonian constraint violation. So far no BSSN code has demonstrated satisfactory performance for this test, and for brevity we do not include BSSN results in the below results.

\subsection{Results}

5.1.1. Results for the Abigel_harm code. For this particular testbed most components of the densitized metric $\bar{g}^{\mu \nu}=\sqrt{-g} g^{\mu \nu}$ have trivial values, the non-trivial ones being

$$
\bar{g}^{y y}=\bar{g}^{z z}=H \text {. }
$$

The original implementation of the Abigel code based upon (B.9) leads to a numerically stable and convergent code, with no high-frequency modes generated. However, as shown by the dramatic growth of the rescaled error plotted in figure 6, the gauge wave excites exponential modes $\bar{g}^{y y}=\bar{g}^{z z}=1-\mathrm{e}^{\lambda t} H, \lambda>0$. This can be understood [21] in terms of solutions of the harmonic system whose densitized metric components are all trivial except for

$$
\bar{g}^{y y}=\bar{g}^{z z}=F(t, x) .
$$

The resulting source term $S^{\mu \nu}$ in (B.9) vanishes except for the components

$$
S^{y y}=S^{z z}=\frac{-F_{t}^{2}+F_{x}^{2}}{F} .
$$




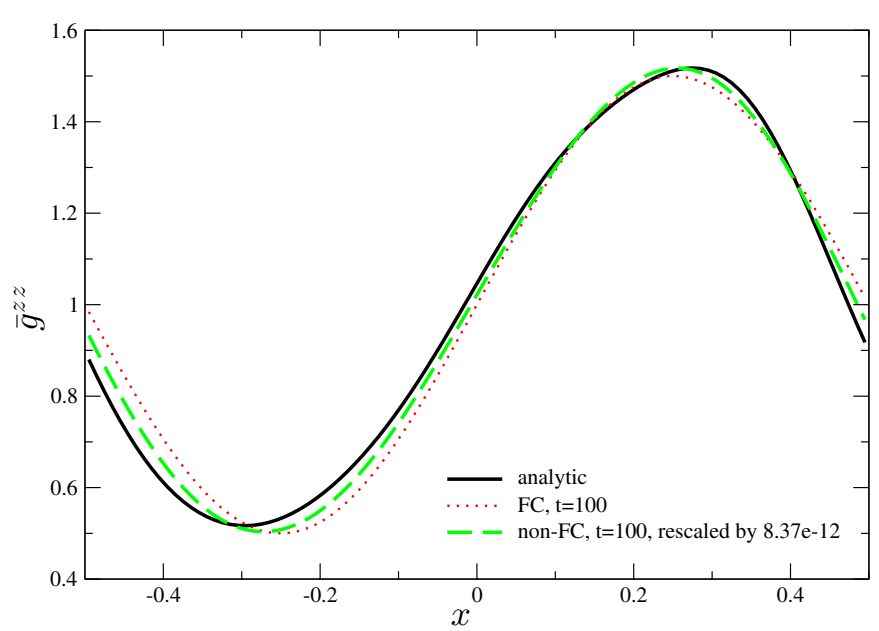

Figure 6. Comparison of code performance between the non-flux-conservative (non-FC) and flux-conservative (FC) versions of the Abigel_harm code, showing graphs of $\bar{g}^{z z}(x)$ at $t=100$ for a gauge wave of amplitude $A=0.5$ on the $\rho=2$ grid. In the non-FC case the graph is rescaled by the average of the plotted function, showing $\bar{g}^{z z} / \operatorname{avg}\left(\bar{g}^{z z}\right) \approx \bar{g}^{z z} / \exp (29.8)$. The good overlap of this rescaled function with the analytic value clearly indicates that the dominant error of the non-FC code is a multiplicative function of $t$. Measurements at $t=100$ for the non-FC code show that logarithm of the spatial average of $\bar{g}^{z z}$ scales roughly as $(\mathrm{d} x)^{2}$, i.e., $\log \left(\operatorname{avg}\left(\bar{g}^{z z}\right)_{\rho=1}\right) \approx 110.8, \log \left(\operatorname{avg}\left(\bar{g}^{z z}\right)_{\rho=2}\right) \approx 29.8, \log \left(\operatorname{avg}\left(\bar{g}^{z z}\right)_{\rho=4}\right) \approx 7.52$, suggesting that the multiplicative error has exponential growth of the form $\exp \left(O\left((\mathrm{~d} x)^{2}\right) \cdot t\right)$.

The PDE for $F(t, x)$, which results from inserting (13) into (B.9), reduces to $\left(-\partial_{t}^{2}+\partial_{x}^{2}\right)$ $\log F=0$, which admits the exponential solutions $F=\mathrm{e}^{\lambda t} H$. These solutions satisfy the harmonic constraints and the reduced harmonic system (B.9), so that they are also solutions of the full Einstein equations. Therefore all codes using harmonic gauge conditions might be expected to excite this mode.

In the case of the Abigel_harm code, these modes were suppressed by building semidiscrete conservation laws into the code which, for the gauge wave initial data, would not be obeyed by the exponential solution. Namely, by writing (B.9) in the flux-conservative form (B.10), the principle part of the resulting equation has vanishing source term, $\tilde{S}^{\mu \nu}=0$, for this test. A summation by parts numerical algorithm then gives rise to the semi-discrete conservation law

$$
\partial_{t} \sum_{I, J, K}\left(g^{t \beta} \partial_{\beta} \bar{g}^{\mu \nu}\right)=0 .
$$

While this is a non-generic result (most spacetimes would give a nonzero source term), building this conservation law into the principal part of the system has proved effective not only in this particular case but in the other Apples with Apples tests considered in this paper, as well as in further proposed tests [21, 23, 24].

As shown in figures 7 and 8 , the flux-conservative code does not develop exponential error modes when running with the original ICN integrator (see [23] for similar results with RK4.) The main source of error is phase error which converges to zero as the grid is refined. In order to further illustrate this point, figures 7 and 8 give test results for both the 1D and 2D versions with amplitudes of $A=0.01,0.1,0.5$. 

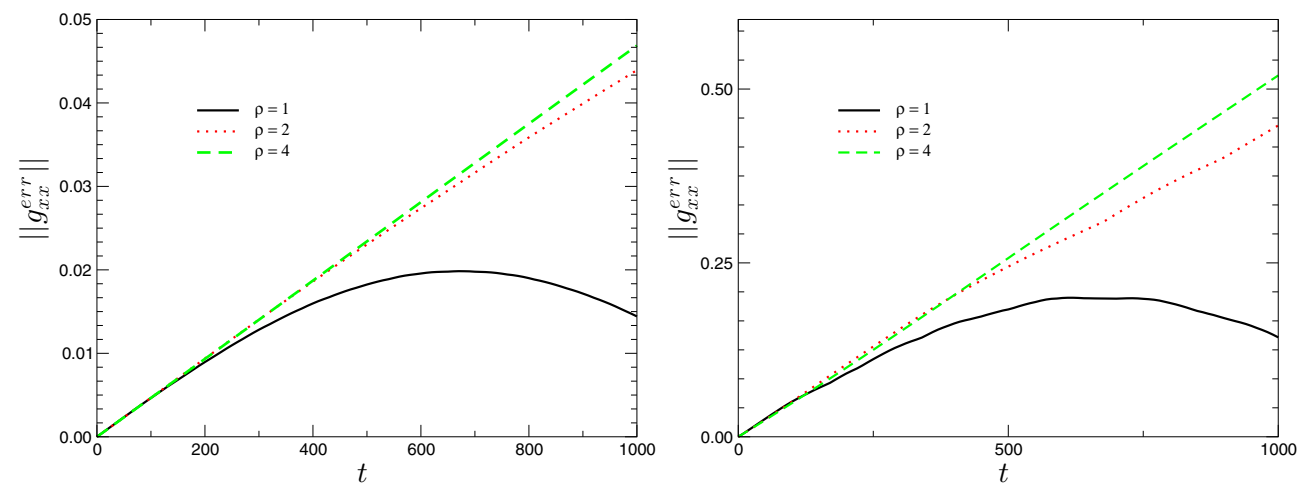

Figure 7. Convergence results for the 1D gauge wave simulation with the Abigel_harm code, for amplitudes of $A=0.01$ (left) and $A=0.1$ (right). The graphs show the $L_{\infty}$-norm of the error in $g_{x x}$, defined as $g_{x x}^{\text {err }}=g_{x x}^{\text {num }}-g_{x x}^{\text {ana }}$ as a function of time, and rescaled by a factor of $1 / \rho^{2}$. As seen from the graphs, the lower amplitude runs give no new information.
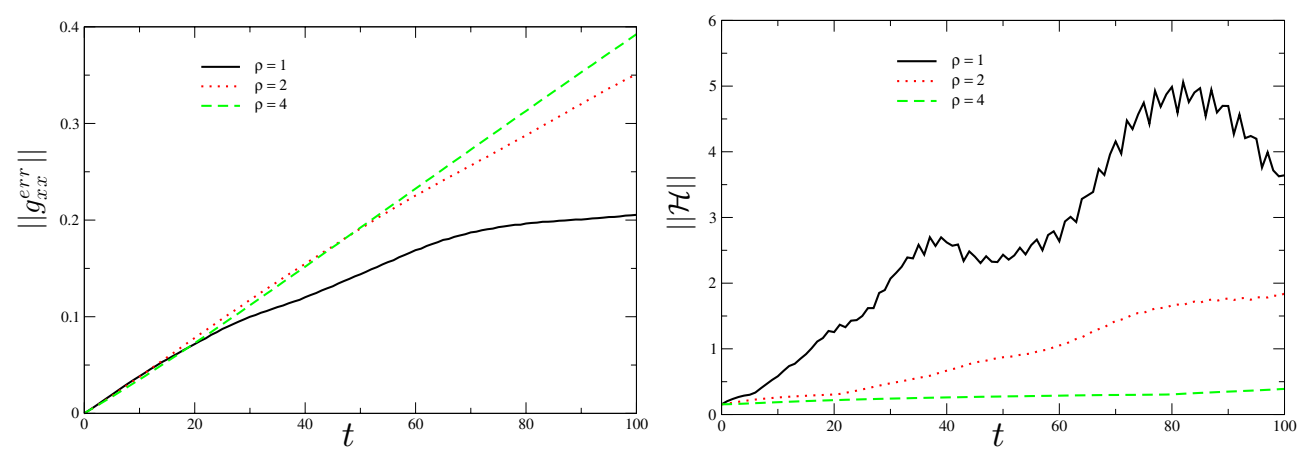

Figure 8. Convergence results for the $2 \mathrm{D}$ gauge wave simulation with the Abigel_harm code, for amplitude $A=0.5$. The left graph shows the $L_{\infty}$-norm of the error in $g_{x x}$, rescaled by a factor of $1 / \rho^{2}$, as a function of time; while the right graph shows the same rescaled error norm for the violation of the Hamiltonian constraint $\mathcal{H}$. For the Abigel_harm code, the vanishing of the Hamiltonian constraint is an algebraic identity, making $\mathcal{H}$ of order roundoff. As a result, the constraint violation is super convergent. The lower amplitude runs revealed no new features.

5.1.2. Results for the HarmNaive system. This naive harmonic system, although weakly hyperbolic, behaves identical to the symmetric hyperbolic Abigel_harm code for this testbed. This can be understood given that the RHS for the mixed spacetime components of the evolution system vanish, i.e.,

$$
\partial_{t} \bar{g}^{i t}=-\partial_{j} \bar{g}^{i j}=0,
$$

which implies that the time-time component of the RHS also vanishes, i.e.,

$$
\partial_{t} \bar{g}^{t t}=-\partial_{j} \bar{g}^{t j}=0 .
$$

The test-results confirm this.

As expected, tests for the ADM-system also behave identically, since the naive harmonic system can be understood as a formulation of the ADM-system in the harmonic gauge. We therefore skip a separate discussion of the ADM-system. 


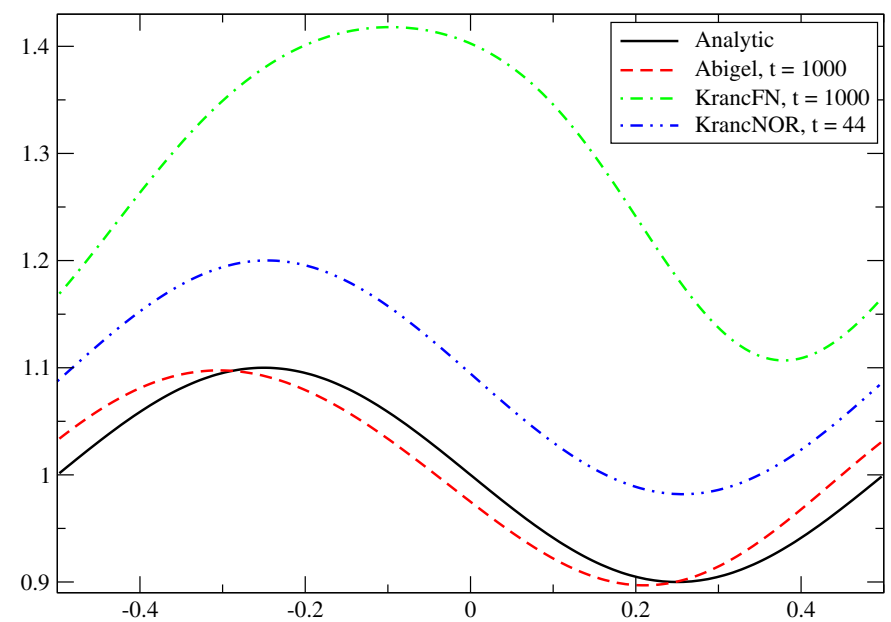

Figure 9. Comparison snapshots of $g_{x x}(x)$ for the $1 \mathrm{D}$ gauge wave with amplitude $A=0.1$ at the end of a run with $\rho=4$ resolution. For the Abigel and KrancFN codes, the run lasts the full 1000 crossing times. The KrancNOR code crashes at $t=44$.

\subsection{Results for the KrancFN and KrancNOR systems}

Besides the harmonic codes, KrancFN was the only other code that was able to run for 1000 crossing times for an amplitude $A=0.1$. At the end of the run, figure 9 shows that long wavelength growth due to the $\mathrm{e}^{\lambda t} H$ instability of the wave amplitude has become appreciable.

The KrancNOR code picks up the $\mathrm{e}^{\lambda t} H$ instability at a faster rate and, although it shows clear second-order convergent at early times, it crashes at $t \approx 44$. The snapshot in figure 9 shows that the error at the end of the run is almost exactly in the $\mathrm{e}^{\lambda t} H$ mode.

\section{Shifted gauge wave testbed}

In the shifted gauge wave test (A.15) we have identified two types of instability [23]. One, which is analogous to the instability of the gauge wave, arises from the $\lambda$-parameter family of vacuum metrics

$$
\mathrm{d} s_{\lambda}^{2}=\mathrm{e}^{\lambda t}\left(-\mathrm{d} t^{2}+\mathrm{d} x^{2}\right)+\mathrm{d} y^{2}+\mathrm{d} z^{2}+H k_{\alpha} k_{\beta} \mathrm{d} x^{\alpha} \mathrm{d} x^{\beta},
$$

which reduces to the shifted gauge wave for $\lambda=0$. The other is an instability peculiar to harmonic (or generalized harmonic) evolution codes, where the Einstein equations are satisfied only indirectly through the harmonic conditions. The metric

$$
\mathrm{d} \hat{s}_{\lambda}^{2}=-\mathrm{d} t^{2}+\mathrm{d} x^{2}+\mathrm{d} y^{2}+\mathrm{d} z^{2}+\left(H-1+\mathrm{e}^{\lambda \hat{t}}\right) k_{\alpha} k_{\beta} \mathrm{d} x^{\alpha} \mathrm{d} x^{\beta},
$$

where

$$
\hat{t}=t-\frac{A d}{4 \pi} \cos \left(\frac{2 \pi(x-t)}{d}\right),
$$

satisfies the reduced harmonic evolution equations (B.9). The simulation of the shifted gauge wave by any evolution code based upon a standard reduction of Einstein's equations to harmonic form can be expected to excite this instability.

The test was developed in conjunction with the Abigel_harm code [23]. For 1D runs with the $\rho=4$ resolution, it was found that the evolution equation (B.10) excited the instability (19) 


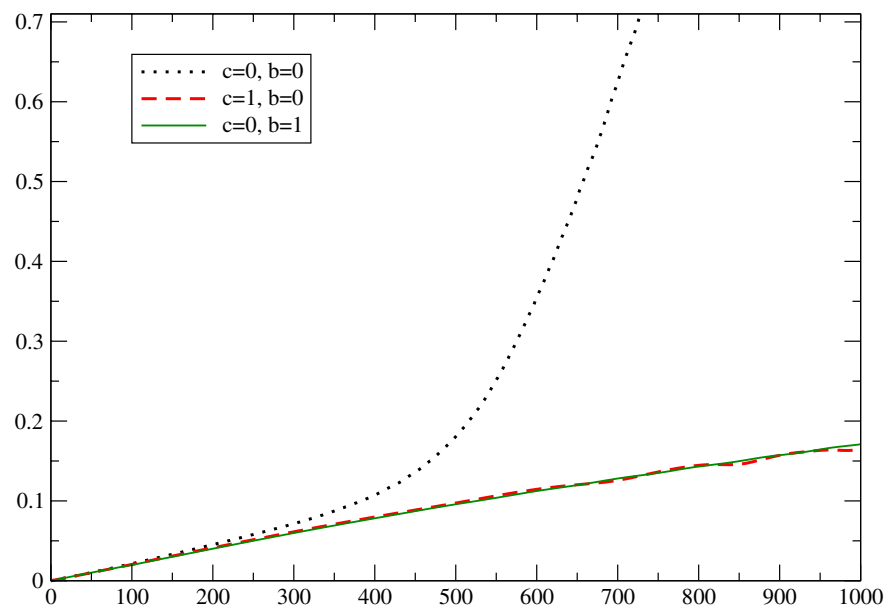

Figure 10. Plots of the $L_{\infty}$ error $\mathcal{E}(t)$ in $g_{x x}$ obtained with the Abigel code for the 1D shifted gauge wave test with amplitude $A=0.5$ and resolution $\rho=4$. Results are compared for the constraint adjustment (23) with $b=1$, the constraint adjustment (24) with $c=1$ and the bare algorithm. The two adjustments show very similar error and both give excellent suppression of the unstable mode excited by the bare algorithm.

on a timescale $t \approx 500$. Further investigation showed that this instability could be suppressed by adjusting (B.10) according to

$$
\tilde{S}^{\mu \nu} \rightarrow \tilde{S}^{\mu \nu}-A^{\mu \nu}
$$

where $A^{\mu \nu}=0$ when the harmonic constraints

$$
C^{\mu}:=-\frac{1}{\sqrt{-g}}\left(\partial_{\nu} \bar{g}^{\mu \nu}-\tilde{H}^{\mu}\right)=0
$$

are satisfied. Particularly effective were the constraint adjustments

$$
A^{\mu \nu}=\frac{b \mathcal{C}^{\alpha} \nabla_{\alpha} t}{e_{\rho \sigma} \mathcal{C}^{\rho} \mathcal{C}^{\sigma}} \mathcal{C}^{\mu} \mathcal{C}^{\nu}, \quad b>0
$$

where $e_{\rho \sigma}$ is the natural metric of signature $(++++)$ associated with the Cauchy slicing, and

$$
A^{\mu \nu}=-\frac{c}{\sqrt{-g}} \mathcal{C}^{\alpha} \partial_{\alpha}\left(\sqrt{-g} g^{\mu \nu}\right), \quad c>0 .
$$

This is exhibited in figure 10, which shows for a run with amplitude $A=0.5$ that these constraint adjustments suppress instabilities for the entire 1000 crossing time duration of the test.

Results for the shifted gauge wave tests are also available from the CVS repository for BSSN codes. In this case, as in the standard gauge wave test, the results are not satisfactory, and suggest further analysis, which is beyond the scope of this paper. Results obtained with the Kranc_BSSN code and a very small value of the dissipation parameter $(\sigma=0.001$, see equation (C.7)) for the medium amplitude $A=0.1$ are shown in figure 11 . While the code shows second-order convergence for several crossing times, rather quickly an instability develops that eventually crashes the code. As expected, the instability develops slower for the lower amplitude $A=0.01$, and faster for $A=0.5$, where the code crashes within roughly one crossing time. Similar results are also available in the CVS repository for the CCATIE code. 

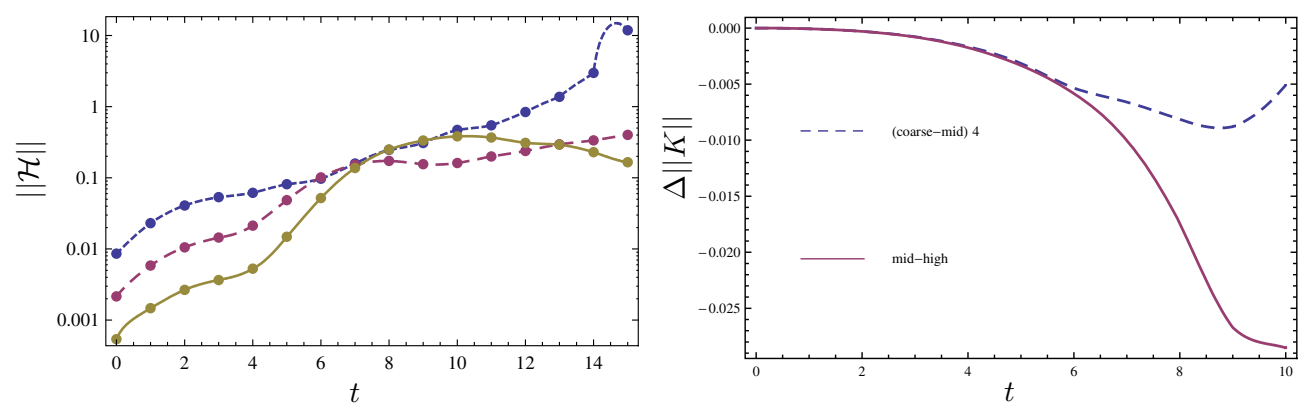

Figure 11. Performance of Kranc_BSSN for the shifted gauge wave with amplitude $A=0.1$ and a dissipation value of $\sigma=0.001$. Left panel. The $L_{2}$-norm of the Hamiltonian constraint plotted versus time for resolutions $\rho=1,2,4$ (short-dashed, long-dashed, full line). Right panel: convergence test for the $L_{2}$-norm of $\operatorname{tr} K$ for resolutions $\rho=1,2,4$. Second-order convergence is lost after a few crossing times.

Results for the shifted gauge wave test have also been obtained [25] using the Caltech-Cornell group's spectral version of a code based upon the Kidder-Scheel-Teukolsky formulation of the Einstein equations [26]. For the 1D test with $A=0.5$, they encountered nonlinear instabilities associated with aliasing after a few crossing times. There are standard filtering techniques to deal with such aliasing problems. By filtering the top $1 / 3$ spectral coefficients, they found that the evolutions could be extended as far as $t=60$, but further improvements by filtering did not seem possible. The group has not yet reported results for their current spectral code which is based upon a generalized harmonic formulation.

\section{Gowdy wave test}

The previous tests involve spacetimes with small curvature. The Gowdy wave test is based upon a strongly curved exact solution for an expanding vacuum universe containing a plane polarized gravitational wave propagating around a 3-torus $T^{3}$ [27]. See [28] for a recent review. The metric has the form

$$
\mathrm{d} s^{2}=t^{-1 / 2} \mathrm{e}^{\lambda / 2}\left(-\mathrm{d} t^{2}+\mathrm{d} z^{2}\right)+t\left(\mathrm{e}^{P} \mathrm{~d} x^{2}+\mathrm{e}^{-P} \mathrm{~d} y^{2}\right),
$$

where $P(t, z)$ and $\lambda(t, z)$ depend periodically on $z$ and the time coordinate $t$ increases as the universe expands, with a cosmological type singularity at $t=0$. The detailed tests specifications given in appendix A.5 were designed so that neither very large nor very small numbers enter in the initial data.

In the expanding direction, the qualitative behavior of the solution is characterized by $P$ slowly decaying to zero while $\lambda$ grows linearly, with both $P$ and $\lambda$ exhibiting gravitational wave oscillations. The linear growth of $\lambda$ leads to exponential growth of $g_{z z}$, so that code accuracy is tested in a harsh situation. This makes evolution with a $3 \mathrm{D}$ code difficult compared with the direct $1 \mathrm{D}$ evolution of $P$ used in numerical studies of the approach to the cosmological singularity [29].

The performance of the various codes in the expanding direction is illustrated by the output for the trace of the extrinsic curvature $K$ shown in figure 12. Although not apparent from the figure, the HarmNaive code crashes abruptly at $t=8$, as might be expected of a weakly hyperbolic system in the nonlinear regime. Even though the analytic value of $K$ is negative and asymptotes to zero with the expansion, short wavelength error in the LS_HyperGR and 


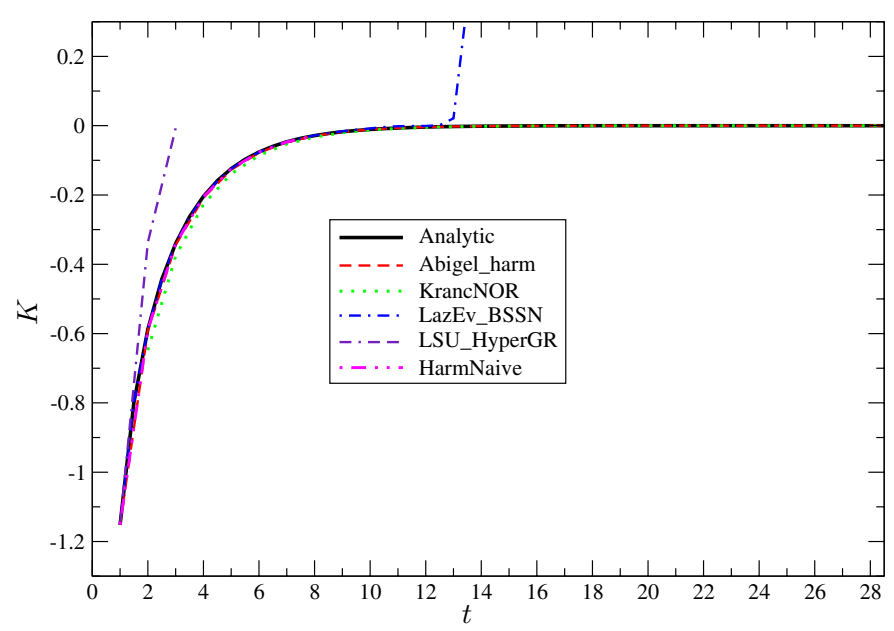

Figure 12. Comparison plots of the trace of the extrinsic curvature $K$ for the polarized Gowdy wave evolved in the expanding direction with the $\rho=4$ resolution. Analytically $K$ is spatially homogeneous; the plots show its maximum value over the numerical grid.

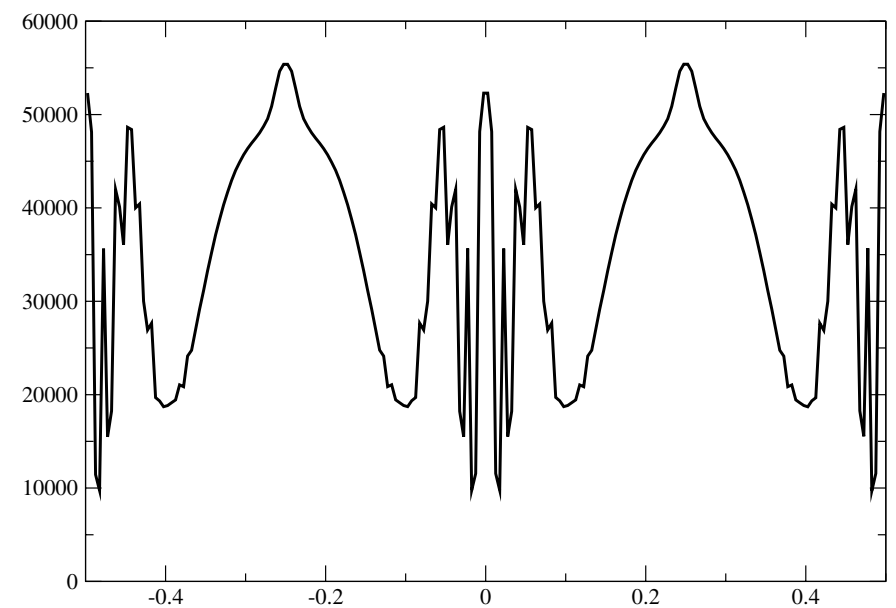

Figure 13. Plot of the error $\mathcal{E}(z)$ in $g_{z z}$ for the polarized Gowdy wave evolved in the expanding direction with $\rho=4$ resolution with the second-order accurate LazEv_BSSN code. The error, plotted at $t=13$ just before the code crashes, shows a large short wavelength component which can be controlled by dissipation.

LazEv_BSSN codes triggers an instability leading to a collapsing mode with $K>0$. This is illustrated for the LazEv_BSSN run in the snapshot of figure 13, which shows the error in $g_{z z}(t, x)$ at $t=13$ just before the run crashes. The superposition of short wavelength error with the long wavelength truncation error from the signal is evident.

Further experiments with the LazEv_BSSN code showed that this short wavelength instability could be controlled by numerical dissipation and that the accuracy could be further improved by using fourth-order finite difference approximations. For the expanding Gowdy test, this is illustrated in the plots of the rescaled error in the left portion of figure 14 which 

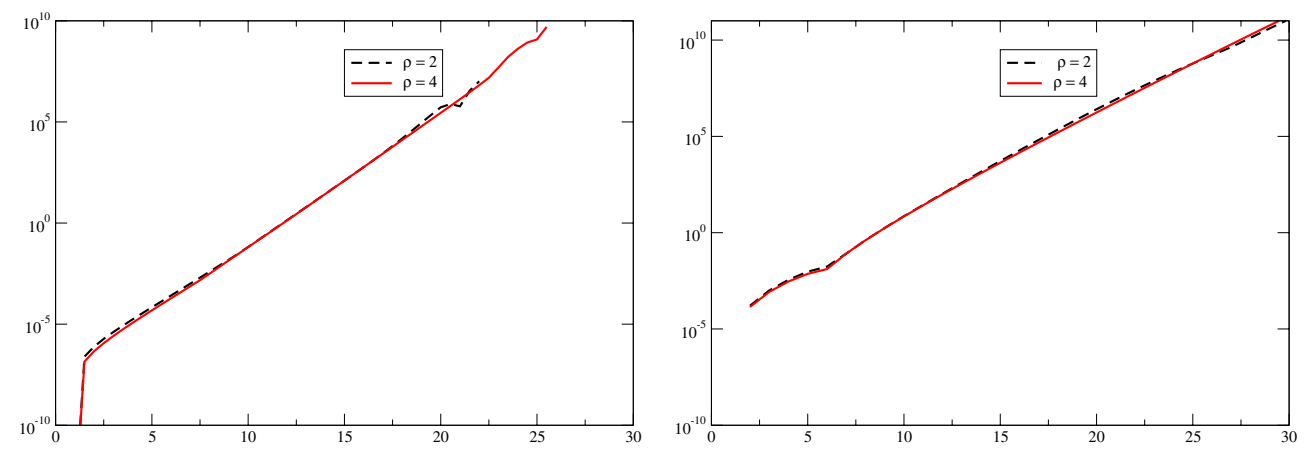

Figure 14. Convergence results for the $L_{\infty}$-norm of the error $\|\mathcal{E}(t)\|$ in $g_{z z}$ (logarithmic scale) for the polarized Gowdy wave evolved in the expanding direction. On the left, the results for the $\rho=2$ resolution have been rescaled by $1 / 16$ for the fourth-order accurate LazEv_BSSN code with dissipation. The results indicate stability and convergence but do not give long-term accuracy. On the right, the error for the $\rho=2$ resolution has been rescaled by $1 / 4$ for the second-order system Abigel_Harm code, again showing stability and convergence. Both codes exhibit roughly the same long-term rate of error growth expected from the exponential growth of $g_{z z}$.

indicate fourth-order convergence. However, the error still exhibits poor long-term accuracy. In the right portion of figure 14, we also display the error in the second-order accurate Abigel_Harm code. Both the second-order and fourth-order codes have approximately the same long-term rate of growth due to the underlying exponential growth in $g_{z z}$.

The Gowdy test is run in both future and past time directions because analytical studies [30] and numerical experiments [22, 31] indicate that the sign of the extrinsic curvature may have important consequences for constraint violation. The subsidiary system governing constraint propagation can lead to unstable departure from the constraint hypersurface. As an example, in a hyperboloidal slicing of Minkowski space with unit lapse and zero shift, the electromagnetic constraint $C=\nabla_{a} E^{a}$ satisfies $C(t)=C(0) \mathrm{e}^{K t}$ when the standard Maxwell evolution equations are satisfied. Thus numerical error can be expected to lead to exponential growth of the constraint for a hyperboloidal foliation with $K>0$. The situation is more complicated in the nonlinear gravitational case but similar instabilities of the system of equations governing the constraints are associated with the extrinsic curvature [30]. A negative value of $K$ (the expanding case) tends to damp constraint violation whereas a positive value (the collapsing case) can trigger constraint violating instabilities.

In the collapsing direction, we perform the runs with a harmonic time slicing to prolong the approach to the singularity, as previously done by Garfinkle [32]. The results for the Hamiltonian constraint for the various codes are shown in figure 15 for the collapsing case. All the codes now show some growth in the Hamiltonian constraint, either of a slow or runaway type. The slow growth, exhibited for example by the Abigel_harm, AEI_CactusEinsteinADM and KrancNOR codes, can be attributed to the analytic constraint instabilities discussed in [30]; the Hamiltonian constraint violation remains small $\left(\approx 10^{-2}\right)$ at the end of the run. The runaway growth exhibited by the LazEv_BSSN code can again be controlled by numerical dissipation. This is demonstrated by the convergence results shown in figure 16 for the fourth-order dissipated version of the code.

The choice of specifications given in appendix A.5 provides a Gowdy testbed capable of good discrimination between different formulations. Results for both the expanding (figure 12) and collapsing (figure 15) directions show a wide spread in the performance 


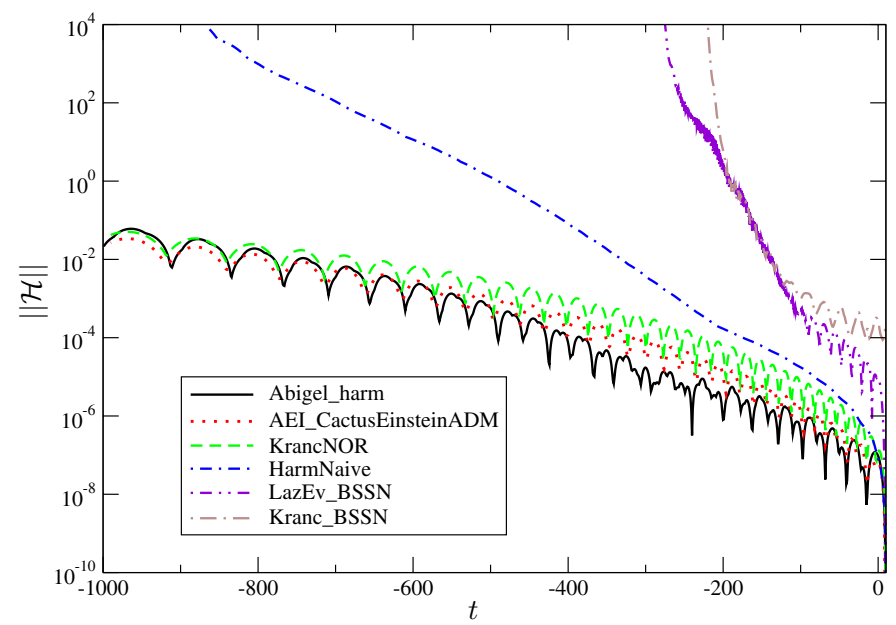

Figure 15. Comparison plot of the $L_{\infty}$-norm of the Hamiltonian constraint versus harmonic time $t$ for the polarized Gowdy wave evolved in the collapsing direction with the $\rho=4$ resolution.

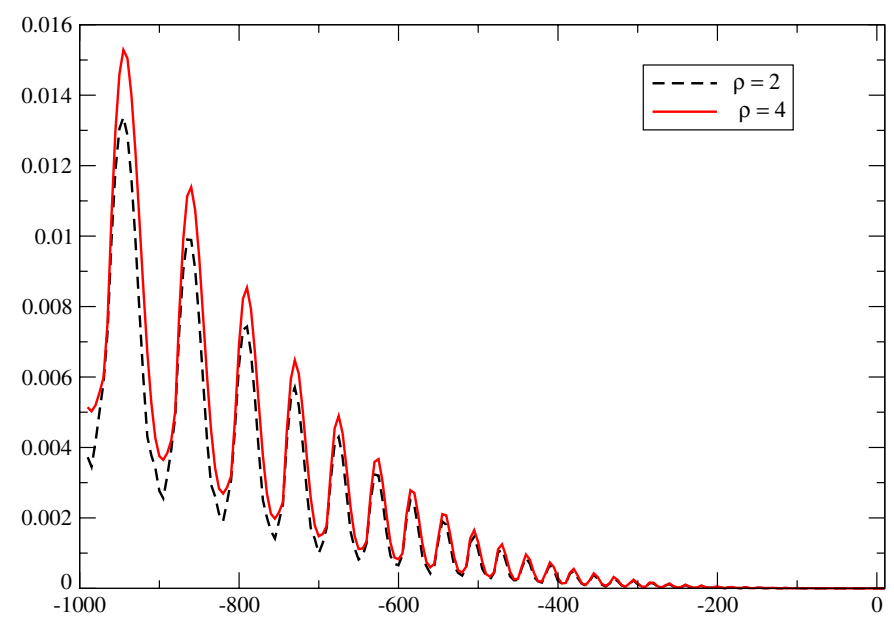

Figure 16. Convergence results for the $L_{\infty}$-norm of the Hamiltonian constraint $\|\mathcal{H}(t)\|$ for the polarized Gowdy wave evolved in the collapsing direction by the fourth-order system LazEv_BSSN code with dissipation. After rescaling the results for the $\rho=2$ by $1 / 16$, they closely match those for the $\rho=4$ resolution. The figure shows stability and convergence of the Hamiltonian constraint up to 1000 crossing times and demonstrates good performance of the LazEv_BSSN code if dissipation is added.

of the different codes. We observe, as in the gauge wave test, that the BSSN-based codes have less satisfactory performance.

\section{Conclusions}

This first round of tests, although modest in scope is a good start at establishing the methods for code verification that have been deemed necessary for any complicated computational 
discipline, such as numerical relativity, to fulfill its scientific potential. As observed by Post and Votta [33] in their study of the verification and validification of large scale computational projects, 'the peer review process in computational science generally does not provide as effective a filter as it does for experiment or theory. Many things that a referee cannot detect could be wrong with a computational science paper.... The few existing studies of error levels in scientific computer codes indicate that the defect rate is about seven faults per 1000 lines of Fortran'. Their observations are especially pertinent for numerical relativity where validation by agreement with experiment is not available.

Several problems have been encountered in the course of this project. One problem was getting prompt response from a broad set of groups with many other pressures. The Apples with Apples workshops were very successful in this regard and were absolutely essential in jump starting and continuing the project. But after the participants dispersed from the workshops, outside pressures led to predictable difficulties. Besides teaching and administrative duties, the overriding scientific pressure in the field has been solving the two black-hole problem and supplying waveforms. This raises a complicated juggling of priorities between black-hole simulations and code verification. In order for code verification to be attractive, the tests have to be useful and the investment in time has to be minimal. This adds emphasis on the need for tests that are simple to carry out and simple to document the results.

Another level of complication in this project arises from the feedback between test design and the analysis of test output. This has led us to improvements in the tests and to their better understanding. In the robust stability test the correct interpretation of results for weakly hyperbolic algorithms required rethinking the proper choice of norm and refinement procedure for judging stability. In the gauge wave tests, the desire for computational efficiency in detecting nonlinear problems at an early time has led us to the adoption of a higher amplitude $A=0.5$ for the test, as opposed to the original specifications $A=0.01$ and $A=0.1$.

The robust stability test is presented as a pass/fail test. For the linear wave test the amplitude and phase errors in the output data for the wave profile provide a good comparison of code performance. For the gauge wave and shifted gauge wave tests, a prime challenge is the suppression of long wavelength nonlinear instabilities in the analytic problem. For the Gowdy test, there were unanticipated shortcomings in the output content that should lend valuable experience in the design of future black-holes tests. Useful benchmarks have been established for the linear wave, gauge wave and Gowdy wave tests, which have revealed clear deficiencies in various codes. Such deficiencies raise a clear alert that it is necessary to apply or recheck other verification techniques, such as convergence tests.

These first round results provide a good basis for proposing new tests. Already, they have prompted addition of the shifted version of the gauge wave test, in which a non-vanishing shift fills a gap in the four original tests for periodic boundary conditions. This test has been useful in developing analytic and numerical techniques for controlling instabilities [23, 25]. A second round of boundary tests based upon the periodic tests have been proposed. The specifications are given on the Alliance website [5]. Results of some of these boundary tests have been reported elsewhere [24, 34]. The next stage is to formulate tests involving black holes.

The code comparisons have proved useful for designing code improvements and for stimulating the use of new numerical techniques. During the course of this work, results of the shifted gauge wave test were key to recognizing the importance of discrete energy and flux conservation for harmonic code performance [23]. The need to carry out the tests with a wide range of formulations has led to the development of symbolic code generation [35]. Although the tests were designed for finite difference codes, they have been adapted and applied to 
pseudo-spectral codes [25]. Further independent studies based upon the tests have played a major part in thesis research [36, 37].

Establishment of the CVS data repository has been an important step in the documentation of test results. Instructions for accessing the data are given at [5]. The CVS directory structure has been significantly streamlined and documented since the beginning of the project. However, the difficulties in completing this analysis of the first round of tests has emphasized the need of a uniform standard for data structures and output. Rather than trying to anticipate a complete list of useful output quantities, it seems more desirable to output the 3-metric and extrinsic curvature at specified times. Then other output quantities can be constructed in post processing. Ideally, this should be done in some standardized way using automated routines and graphical interfaces. All of this would require considerable infrastructure to provide hardware for data storage and software for processing. This is one of the important matters that will be presented for discussion at future Alliance meetings.

\section{Acknowledgments}

We are grateful to UNAM in Mexico City, to the University of Cordoba and to the Center for Computation \& Technology at Louisiana State University for their hospitality in conducting AwA workshops. MCB, SH, CL, ES and JW gratefully acknowledge hospitality of the Albert Einstein Institute, and $\mathrm{SH}$ of the University of the Balearic Islands. We benefited from discussions with Miguel Alcubierre, Adel Benlagra, Carles Bona, Mihaela Chirvasa, Helmut Friedrich, Ian Hawke, Frank Herrmann, Carlos Palenzuela, Oscar Reula, Krzysztof Roszkowski, Marcelo Salgado, Hisa-aki Shinkai, Manuel Tiglio and Tilman Vogel. The work of MCB and JW was supported by NSF grant PH-0553597 to the University of Pittsburgh. YZ was supported by NFS grants PHY-0722315, PHY-0722703, PHY-0714388 and PHY-653303, and by the NASA Center for Gravitational Wave Astronomy at the University of Texas at Brownsville (NAG5-13396). DA has been supported by the Spanish Ministero de Education y Ciencia, projects FPA-2007-60220 and HA2007-0042, SH has been supported by DAAD through a PPP-project with the University of the Balearic Islands and by DFG through the SFB/TR7. S Husa is a VESF fellow of the European Gravitational Observatory (EGO).

\section{Appendix A. Revised testbed specifications}

We present here the updated specifications for the five standardized testbeds. For each test we provide the 4-metric of the spacetime, except for the robust stability test where only the initial Cauchy data are specified. This determines the 3-metric $h_{\mu \nu}=g_{\mu \nu}+n_{\mu} n_{v}$ (where $n_{\mu}$ is the future directed unit normal to the Cauchy hypersurface) and the extrinsic curvature $K_{\mu \nu}$. We use the convention $K_{\mu \nu}=-h_{\mu}^{\rho} \nabla_{\nu} n_{\rho}$ for which the trace $K$ is negative for an expanding cosmology. In all cases, the evolution takes place in a fixed rectilinear coordinate domain with periodic boundary conditions, i.e. a 3-torus. The identified 'boundaries' in the 3-torus picture are located a half step from the first and last grid points along each axis.

Even though we are concerned with three-dimensional codes, for tests with only onedimensional features in the $x$-direction it is efficient to use the minimum number of grid points in the trivial $y$ - and $z$-directions, i.e. to run the test in a long channel rather than a cube. For standard second-order finite differencing this implies that we use 3 or 4 points in those directions. For all such $1 \mathrm{D}$ tests, the evolution domain is

$$
x \in[-0.5,+0.5], \quad y=0, \quad z=0,
$$


with grid

$x=-0.5+\left(n-\frac{1}{2}\right) \mathrm{d} x, \quad n=1 \ldots 50 \rho, \quad \mathrm{d} x=1 /(50 \rho), \quad \rho \in \mathbb{Z}$.

(In the Gowdy wave test, the grid is aligned with the $z$-direction.) The $2 \mathrm{D}$ tests have evolution domain

$$
x \in[-0.5 ;+0.5] \quad y \in[-0.5 ;+0.5], \quad z=0
$$

with both the $x$ and $y$ grids satisfying (A.2). The parameter $\rho$ allows for grid refinement. The coarsest $\rho=1$ grid is useful only for debugging. Convergence tests should be made with $\rho=2$ and $\rho=4$, with benchmarks for norms, constraints, etc provided by $\rho=4$.

We have dropped the original requirement that the tests be run with an iterative-CrankNicholson algorithm since Runge-Kutta time integrators have since proved to be more effective and have been commonly adopted. For each test, the size of the timestep $\mathrm{d} t$ is given in terms of the grid size to lie within the CFL limit for an explicit evolution algorithm. (For some codes this may be inappropriate and some equivalent choice of time step should be made.) A final time $T$, and intermediate times for data output, are specified for each test. They are chosen to incorporate all useful features of the test without prohibitive computational expense. Except for the robust stability test, it is important to calculate the convergence rate of the numerical error. Additional output variables might be essential to assess the performance of a particular formulation.

\section{A.1. Robust stability testbed}

The 3-metric is initialized as $h_{i j}=\delta_{i j}+\epsilon_{i j}$, where $\epsilon_{i j}$ are independent random numbers at each grid point. All other evolution variables are initialized in the same way. The amplitude of the random noise is scaled with the grid as

$$
\epsilon \in\left(-10^{-10} / \rho^{2},+10^{-10} / \rho^{2}\right) .
$$

The range of the random numbers ensures that $\epsilon^{2}$ effects are below round-off accuracy so that the evolution remains in the linear domain unless instabilities arise.

The timestep is specified to be $\mathrm{d} t=\mathrm{d} x / 10=0.002 / \rho$. The use of four distinct gridpoints in the $y$-and $z$-directions allows for instabilities associated with the checkerboard mode.

The test should be run until one is confident that dissipation effects do not cloud the result. Without artificial dissipation, a run time of one crossing time, using output at every time step, is usually sufficient. This corresponds to $500 \rho$ time steps. The test is passed if the norm satisfies the inequality (11) for all resolutions, for some fixed choice of constants $A$ and $K$. Appropriate norms for both first- and second-order systems are recommended in [11] and are publicly available as Cactus thorns [38].

\section{A.2. Linear wave testbed}

The initial 3-metric and extrinsic curvature $K_{i j}$ are given by a transverse, trace-free perturbation with components

$$
\mathrm{d} s^{2}=-\mathrm{d} t^{2}+\mathrm{d} x^{2}+(1+H) \mathrm{d} y^{2}+(1-H) \mathrm{d} z^{2},
$$

where

$$
H=A \sin \left(\frac{2 \pi(x-t)}{d}\right)
$$


This describes a linearized plane wave traveling in the $x$-direction. The wavelength is set to $d=1$ to match the periodicity of the evolution domain. The metric has lapse $\alpha=1$ and shift $\beta^{i}=0$. The nontrivial components of extrinsic curvature are

$$
K_{y y}=-\frac{1}{2} \partial_{t} H, \quad K_{z z}=\frac{1}{2} \partial_{t} H .
$$

In order to test two-dimensional effects, the rotation

$$
x=\frac{1}{\sqrt{2}}\left(x^{\prime}-y^{\prime}\right), \quad y=\frac{1}{\sqrt{2}}\left(x^{\prime}+y^{\prime}\right) .
$$

leads to a wave propagating along a diagonal. The resulting metric is a function of

$$
\sin \left(\frac{2 \pi\left(x^{\prime}-y^{\prime}-t \sqrt{2}\right)}{d^{\prime}}\right), \quad \text { where } \quad d^{\prime}=d \sqrt{2} .
$$

To obtain the required periodicity of the evolution domain, we set $d=1$ in the 1D simulation and $d^{\prime}=1$ in the diagonal simulation. The test should be run in both axis-aligned and diagonal form.

The test is performed with amplitude $A=10^{-8}$, so that quadratic terms are of the order of numerical round-off. The time step is set to $\mathrm{d} t=\mathrm{d} x / 4=0.005 / \rho$ As in the gauge wave case, the $1 \mathrm{D}$ evolution is carried out for $T=1000$ crossing times, i.e. $2 \times 10^{5} \rho$ time steps, with output every ten crossing times. The $2 \mathrm{D}$ diagonal runs are carried out for $T=100$, with output every crossing time. The output quantities are the $L_{\infty}$ - and $L_{2}$-norms, the maxima and minima, and profiles along the $x$-axis through the center of the grid of $g_{y y}, g_{z z}$, Hamiltonian constraint; and the $L_{\infty}$ error norm for $g_{z z}$ (measuring the difference from the exact solution).

\section{A.3. Gauge wave testbed}

The test is based upon the 4-metric

$$
\mathrm{d} s^{2}=(1-H)\left(-\mathrm{d} t^{2}+\mathrm{d} x^{2}\right)+\mathrm{d} y^{2}+\mathrm{d} z^{2},
$$

with $H$ given by (A.6), which is obtained from the Minkowski metric $\mathrm{d} s^{2}=-\mathrm{d} \hat{t}^{2}+\mathrm{d} \hat{x}^{2}+$ $\mathrm{d} \hat{y}^{2}+\mathrm{d} \hat{z}^{2}$ by the transformation

$$
\begin{aligned}
& \hat{t}=t-\frac{A d}{4 \pi} \cos \left(\frac{2 \pi(x-t)}{d}\right), \\
& \hat{x}=x+\frac{A d}{4 \pi} \cos \left(\frac{2 \pi(x-t)}{d}\right), \\
& \hat{y}=y, \\
& \hat{z}=z .
\end{aligned}
$$

This describes a sinusoidal gauge wave of amplitude $A$ propagating along the $x$-axis. The extrinsic curvature is

$$
\begin{aligned}
& K_{x x}=\frac{\partial_{t} H}{2 \sqrt{1-H}}=-\frac{\pi A}{d} \frac{\cos \left(\frac{2 \pi(x-t)}{d}\right)}{\sqrt{1-A \sin \left(\frac{2 \pi(x-t)}{d}\right)}}, \\
& K_{i j}=0 \quad \text { otherwise. }
\end{aligned}
$$

As for the linear wave, the rotation (A.8) leads to wave propagation along a diagonal with periodic boundary conditions.

The gauge wave is run with amplitude $A=0.5$. The time coordinate $t$ in the metric (A.10) is harmonic and the gauge speed is the speed of light. The time step is set to 
$\mathrm{d} t=\mathrm{d} x / 4=0.005 / \rho$. The $1 \mathrm{D}$ evolution is carried out for $T=1000$ crossing times, i.e. $2 \times 10^{5} \rho$ time steps (or until the code crashes), with output every ten crossing times. The 2D diagonal runs are carried out for $T=100$, with output every crossing time.

Output consists of the $L_{\infty}$ - and $L_{2}$-norms, the maxima and minima, and profiles along the $x$-axis through the center of the grid $(y=z=0)$ of $g_{x x}, \alpha, \operatorname{tr}(K)$ and the Hamiltonian constraint; and the $L_{2}$ error-norm for $g_{x x}$.

\section{A.4. The shifted gauge wave test}

The shifted gauge wave is obtained from the Minkowski metric $\mathrm{d} s^{2}=-\mathrm{d} \hat{t}^{2}+\mathrm{d} \hat{x}^{2}+\mathrm{d} \hat{y}^{2}+\mathrm{d} \hat{z}^{2}$ by the harmonic coordinate transformation

$$
\begin{aligned}
& \hat{t}=t-\frac{A d}{4 \pi} \cos \left(\frac{2 \pi(x-t)}{d}\right), \\
& \hat{x}=x-\frac{A d}{4 \pi} \cos \left(\frac{2 \pi(x-t)}{d}\right), \\
& \hat{y}=y, \\
& \hat{z}=z
\end{aligned}
$$

which leads to the Kerr-Schild metric

$$
\mathrm{d} s^{2}=-\mathrm{d} t^{2}+\mathrm{d} x^{2}+\mathrm{d} y^{2}+\mathrm{d} z^{2}+H k_{\alpha} k_{\beta} \mathrm{d} x^{\alpha} \mathrm{d} x^{\beta}
$$

where

$$
k_{\alpha}=-\partial_{\alpha}(t-x)
$$

and $H$ is again given by (A.6). The extrinsic curvature is

$$
\begin{aligned}
& K_{x x}=\frac{\partial_{t} H}{2 \sqrt{1+H}}, \\
& K_{i j}=0 \quad \text { otherwise. }
\end{aligned}
$$

This metric describes a shifted gauge wave of amplitude $A$ propagating along the $x$-axis. The coordinate transformation (A.8) rotates the propagation direction to the diagonal.

The shifted gauge wave test is run in a harmonic gauge with amplitude $A=0.5$ in both $1 \mathrm{D}$ form and diagonal 2D form. As in the linear wave test, for the required periodicity we set $d=1$ in the $1 \mathrm{D}$ simulations and $d^{\prime}=1$ in the $2 \mathrm{D}$ simulations. We set the timestep $\mathrm{d} t=\mathrm{d} x / 4=0.005 / \rho$. The $1 \mathrm{D}$ evolution is carried out for $T=1000$ crossing times, i.e. $2 \times 10^{5} \rho$ time steps (or until the code crash). The $2 \mathrm{D}$ runs are carried out for $T=100$.

Output data consist of the profiles along the $x$-axis through the center of the grid ( $y=z=0$ ) of $g_{t t}, g_{x t}$ and $g_{x x}$, the $L_{2^{-}}$and $L_{\infty}$-norms of the error and of the Hamiltonian constraint.

\section{A.5. Polarized Gowdy wave testbed}

The polarized Gowdy metrics describe an expanding, toroidal universe containing plane polarized gravitational waves with metric

$$
\mathrm{d} s^{2}=t^{-1 / 2} \mathrm{e}^{\lambda / 2}\left(-\mathrm{d} t^{2}+\mathrm{d} z^{2}\right)+t \mathrm{e}^{P} \mathrm{~d} x^{2}+\mathrm{e}^{-P} \mathrm{~d} y^{2}
$$

where $\lambda$ and $P$ are functions of $z$ and $t$ only and are periodic in $z$. The universe expands as $t$ increases. The test is carried out in both the collapsing and expanding situations. The metric is singular at $t=0$. 
The Einstein equations reduce to a single evolution equation

$$
P_{, t t}+t^{-1} P_{, t}-P_{, z z}=0
$$

and the constraint equations

$$
\lambda_{, t}=t\left(P_{, t}^{2}+P_{, z}^{2}\right)
$$

and

$$
\lambda_{, z}=2 t P_{, z} P_{, t} \text {. }
$$

The test is based upon the particular solution to (A.20)

$$
P=J_{0}(2 \pi t) \cos (2 \pi z),
$$

where $J_{n}$ are Bessel functions. The metric and extrinsic curvature are

$$
\begin{aligned}
& g_{x x}=t \mathrm{e}^{P}, \quad g_{y y}=t \mathrm{e}^{-P}, \quad g_{z z}=t^{-1 / 2} \mathrm{e}^{\lambda / 2}, \\
& K_{x x}=-\frac{1}{2} t^{1 / 4} \mathrm{e}^{-\lambda / 4} \mathrm{e}^{P}\left(1+t P_{, t}\right), \\
& K_{y y}=-\frac{1}{2} t^{1 / 4} \mathrm{e}^{-\lambda / 4} \mathrm{e}^{-P}\left(1-t P_{, t}\right), \\
& K_{z z}=\frac{1}{4} t^{-1 / 4} \mathrm{e}^{\lambda / 4}\left(t^{-1}-\lambda_{, t}\right),
\end{aligned}
$$

with

$$
\operatorname{tr} K=-\frac{1}{4} t^{1 / 4} \mathrm{e}^{-\lambda / 4}\left(3 t^{-1}+\lambda_{, t}\right) .
$$

The shift vanishes and the lapse is

$$
\alpha=\sqrt{g_{z z}}=t^{-1 / 4} \mathrm{e}^{\lambda / 4} .
$$

For the choice (A.23), the constraints (A.21), (A.22) yield

$$
\begin{array}{r}
\lambda=-2 \pi t J_{0}(2 \pi t) J_{1}(2 \pi t) \cos ^{2}(2 \pi z)+2 \pi^{2} t^{2}\left[J_{0}^{2}(2 \pi t)+J_{1}^{2}(2 \pi t)\right] \\
-\frac{1}{2}\left\{(2 \pi)^{2}\left[J_{0}^{2}(2 \pi)+J_{1}^{2}(2 \pi)\right]-2 \pi J_{0}(2 \pi) J_{1}(2 \pi)\right\} .
\end{array}
$$

While $P$ slowly decays to zero, $\lambda$ undergoes linear growth due to the cosmological expansion, and both $P$ and $\lambda$ exhibit gravitational wave oscillations.

The velocity of light is constant in the coordinates chosen in (A.19) so that, with a fixed spatial discretization $\mathrm{d} z$, the Courant condition is consistent with a fixed timestep $\mathrm{d} t$. This makes the gauge (A.19) convenient for evolving in the expanding direction by choosing the initial data from the exact solution at $t=1$, which yields data of order unity.

In the backward in time evolution, we choose a harmonic time slicing $\tau$ which only asymptotically reaches the singularity. Starting with the metric (A.19), the slicing is obtained by a transformation $t=F(\tau)$, where the harmonic condition $\square \tau=0$ implies $F(\tau)=k \mathrm{e}^{c \tau}$. In order to start the collapse slowly, the free constants $c$ and $k$ are chosen so that the new lapse satisfies $\hat{\alpha}=1$ at the initial time $t=t_{0}$. This is accomplished by picking $t_{0}$ for which $J_{0}\left(2 \pi t_{0}\right)=0$ so that (A.28) implies $\hat{\alpha}$ is independent of $z$. Using

$$
\tau_{0}=\frac{1}{c} \ln \left(\frac{t_{0}}{k}\right), \quad \lambda\left(k \mathrm{e}^{c \tau_{0}}, z\right)=\lambda_{0}
$$

we obtain

$$
\hat{\alpha}_{0}=c t_{0}^{3 / 4} \mathrm{e}^{\lambda_{0} / 4}
$$

Given our requirement $\hat{\alpha}_{0}=1$, and choosing $t_{0}=\tau_{0}$, i.e. $F\left(\tau_{0}\right)=\tau_{0}$, we get

$$
c=t_{0}^{-3 / 4} \mathrm{e}^{-\lambda_{0} / 4}, \quad k=t_{0} \mathrm{e}^{-c t_{0}} .
$$


We choose a particular value of $t_{0}$ such that the initial slice is far from the cosmological singularity, but not so far that we have to deal with extremely large numbers by picking the 20th zero of the Bessel function $J_{0}\left(2 \pi t_{0}\right)$, which yields $t_{0} \sim 9.8753205829098$, corresponding to

$$
c \sim 0.0021195119214617, \quad k \sim 9.6707698127638 .
$$

The time step is set to $\mathrm{d} t=\mathrm{d} z / 4=0.005 / \rho$ with run time $T=1000$ or until code crash. Output consists of the $L_{\infty^{-}}$and $L_{2}$-norms, the maxima and minima, and profiles along the $z$-axis through the center of the grid of $g_{z z}, \alpha, \operatorname{tr}(K)$ and the Hamiltonian constraint. We output norms every crossing time, and profiles either every ten crossing times or once per crossing time, depending on the behavior of the simulation. We also output the $L_{\infty}$ error norms of the difference from the exact solution for $g_{x x}$ and $g_{z z}$ for the expanding direction.

\section{Appendix B. Code descriptions}

\section{B.1. Standard ADM: Kranc_FreeADM, and AEI_CactusEinsteinADM codes}

The formulation of the Einstein equation by Arnowitt, Deser and Misner (ADM) [39] provides a standard notion for 'evolving' spacetime as an initial-value problem in general relativity, which was initially presented in a Hamiltonian context. What is referred to as a 'standard ADM' system in the numerical relativity community is a reformulation due to York [40], which one obtains by $(3+1)$-decomposition of the Einstein tensor (as opposed to $(3+1)$-decomposition of the Ricci tensor in the original ADM version), or equivalently by adding appropriate constraint terms to the evolution equations. As pointed out by Frittelli [41], York's 'standard ADM' system does in particular have nicer properties regarding the constraint propagation system. This system is particularly simple, has a long history in numerical relativity and exhibits some typical problems. We therefore use it as the starting point for our numerical comparisons. The evolution equations are

$$
\begin{aligned}
& \partial_{t} \gamma_{i j}=-2 \alpha K_{i j}+\nabla_{i} \beta_{j}+\nabla_{j} \beta_{i} \\
& \partial_{t} K_{i j}=\alpha R_{i j}^{(3)}+\alpha K K_{i j}-2 \alpha K_{i k} K_{j}^{k}-\nabla_{i} \nabla_{j} \alpha+\left(\nabla_{i} \beta^{k}\right) K_{k j}+\left(\nabla_{j} \beta^{k}\right) K_{k i}+\beta^{k} \nabla_{k} K_{i j}
\end{aligned}
$$

and the constraint equations are

$$
\begin{aligned}
& \mathcal{H}=\mathcal{H}^{A D M}:=R^{(3)}+K^{2}-K_{i j} K^{i j}, \\
& \mathcal{M}_{i}=\mathcal{M}_{i}^{A D M}:=\nabla_{j} K^{j}{ }_{i}-\nabla_{i} K,
\end{aligned}
$$

where $\left(\gamma_{i j}, K_{i j}\right)$ are the induced 3-metric and the extrinsic curvature, $\left(\alpha, \beta_{i}\right)$ are the lapse function and the shift covector, $\nabla_{i}$ is the three-dimensional covariant derivative and $R_{i j}^{(3)}$ is the three-dimensional Ricci tensor associated with $\gamma_{i j}$.

We have tested two implementations of the standard ADM system, the code AEI_CactusEinsteinADM, which is freely available via the website [42], and Kranc_FreeADM which is based on the Cactus Toolkit [42] and Kranc software [35]. AEI_CactusEinsteinADM uses a hardcoded ICN time update scheme (see, e.g., [11]), whereas Kranc_FreeADM uses a method of lines (MoL) approach based on the CactusMoL thorn (in practice, RK3, RK4 and ICN (see, e.g., [11]) have also been used, as indicated). In all of these codes, spatial partial derivatives are reduced to partial derivatives of the 3-metric, i.e., all expressions such as Christoffel symbols are expanded out. Due to the absence of first-order variables, no further ambiguities arise. Centered second- and fourth-order discretization is used (see 
appendix C.1), and third-order Kreiss-Oliger dissipation is optionally applied to all variables (see appendix C.2).

The hyperbolicity of the ADM free evolution scheme has been analyzed and found to be weakly hyperbolic with the type of gauge conditions that we use [11]. Since many of our tests are essentially 1D tests, where ADM yields good results, we have also analyzed the hyperbolicity of ADM in 1D. For simplicity of presentation we restrict ourselves to the linearized case. Assuming propagation in the $x$-direction we obtain the following evolution equations. For the off-diagonal components,

$$
\partial_{t} \gamma_{y z}=2 K_{y z}, \quad \partial_{t} K_{y z}=\partial_{x x} \gamma_{y z} / 2, \quad \partial_{t} K_{x y}=0, \quad \partial_{t} K_{x z}=0 .
$$

The evolution equations for $\gamma_{x y}$ and $\gamma_{x z}$ are analogous to the evolution equation for $\gamma_{y z}$. The fact that the evolution equations for $K_{x y}$ and $K_{x z}$ are trivial renders the evolution system for the off-diagonal components weakly hyperbolic (see, e.g., [11]). For the diagonal components,

$$
\begin{aligned}
& \partial_{t} \gamma_{i i}=2 K_{i i} \quad(i=x, y, z), \\
& \partial_{t} K_{x x}=\partial_{x x} \alpha+\frac{1}{2} \partial_{x x}\left(\gamma_{y y}+\gamma_{z z}\right), \\
& \partial_{t} K_{j j}=\frac{1}{2} \partial_{x x} \gamma_{j j} \quad(j=y, z) .
\end{aligned}
$$

Considering for simplicity the densitized lapse case, $\alpha=\sqrt{\gamma}$, the evolution equation for $K_{x x}$ becomes

$$
\partial_{t} K_{x x}=\frac{1}{2} \partial_{x x} \gamma_{x x}+\partial_{x x}\left(\gamma_{y y}+\gamma_{z z}\right)
$$

and one finds that the diagonal subsystem is only weakly hyperbolic. However, within the subclasses of gauge wave $\left(\gamma_{y y}=\gamma_{z z}=0\right)$ or linear wave $\left(\gamma_{x x}=0\right)$ data, the 1D ADM system corresponds to copies of the $1 \mathrm{D}$ wave equation and is therefore well-posed.

\section{B.2. Abigel_harm}

The Abigel code developed in Pittsburgh is based upon a symmetric hyperbolic formulation of the Einstein equations using generalized harmonic coordinates satisfying the curved space wave equation

$$
\square x^{\alpha}=\frac{1}{\sqrt{-g}} \partial_{\mu}\left(\sqrt{-g} g^{\mu \nu} \partial_{\nu} x^{\alpha}\right)=\frac{1}{\sqrt{-g}} \tilde{H}^{\alpha}\left(x^{\beta}, g_{\rho \sigma}\right),
$$

where $\tilde{H}^{\alpha}$ are harmonic source terms. The original version of the evolution equations was [43]

$$
\bar{g}^{\alpha \beta} \partial_{\alpha} \partial_{\beta} \bar{g}^{v \mu}=S^{\mu \nu},
$$

where the left-hand side is the principle part and the right-hand side contains nonlinear firstderivative terms. Here $\bar{g}^{\mu \nu}=\sqrt{-g} g^{\mu \nu}$, with $g=\operatorname{det}\left(g_{\mu v}\right)=\operatorname{det}\left(\bar{g}^{\mu \nu}\right)$, and the harmonic constraints $\partial_{\nu} \bar{g}^{\mu \nu}=\tilde{H}^{\mu}$ are used in the Einstein equations to eliminate second derivatives in the source terms $S^{\mu \nu}$. For further details concerning the formulation and its implementation see [43].

The code with which the tests were performed was constructed by rewriting (B.9) in the flux conservative form

$$
\partial_{\alpha}\left(g^{\alpha \beta} \partial_{\beta} \bar{g}^{\mu \nu}\right)=\tilde{S}^{\mu \nu},
$$

and reducing it to the first order in time form

$$
\partial_{t} \bar{g}^{\mu \nu}=-\frac{\bar{g}^{t i}}{\bar{g}^{t t}} \partial_{i} \bar{g}^{\mu \nu}+\frac{\sqrt{-g}}{\bar{g}^{t t}} Q^{\mu \nu}
$$




$$
\begin{aligned}
\partial_{t} Q^{\mu \nu} & =-\partial_{i}\left(g^{i j} \partial_{j} \bar{g}^{\mu \nu}+g^{i t} \partial_{t} \bar{g}^{\mu \nu}\right)+\tilde{S}^{\mu \nu} \\
& =-\partial_{i}\left[\left(g^{i j}-\frac{g^{t i} g^{t j}}{g^{t t}}\right) \partial_{j} \bar{g}^{\mu \nu}\right]-\partial_{i}\left(\frac{g^{i t}}{g^{t t}} Q^{\mu \nu}\right)+\tilde{S}^{\mu \nu} \\
& =-\partial_{i}\left(h^{i j} \partial_{j} \bar{g}^{\mu \nu}\right)-\partial_{i}\left(\frac{g^{i t}}{g^{t t}} Q^{\mu \nu}\right)+\tilde{S}^{\mu \nu}
\end{aligned}
$$

in terms of the evolution variables $\left(\bar{g}^{\mu \nu}, Q^{\mu \nu}\right)$, where

$$
Q^{\mu \nu}=g^{t \alpha} \partial_{\alpha} \bar{g}^{\mu \nu}
$$

and $h^{i j}=g^{i j}-g^{i t} g^{j t} / g^{t t}$ is the spatial 3-metric. Centered derivatives are used to finite difference (B.11) and the source terms $\tilde{S}^{\mu \nu}$ in (B.14). The remaining part of equation (B.14) is finite-differenced as follows:

$$
\begin{aligned}
& g_{[I+1 / 2, J, K]}^{\alpha \beta}=\frac{A_{+x} \bar{g}_{[I, J, K]}^{\alpha \beta}}{\sqrt{-A_{+x} g_{[I, J, K]}}}+\mathcal{O}\left(\Delta^{2}\right) \\
& h_{[I+1 / 2, J, K]}^{i j}=g_{[I+1 / 2, J, K]}^{i j}-\frac{g_{[I+1 / 2, J, K]}^{t i} g_{[I+1 / 2, J, K]}^{t j}}{g_{[I+1 / 2, J, K]}^{t t}} \\
& \partial_{x}\left(h^{x x} \partial_{x} \bar{g}^{\mu \nu}\right)_{[I, J, K]}=D_{-x}\left(h_{[I+1 / 2, J, K]}^{x x} D_{+x} \bar{g}_{[I, J, K]}^{\mu \nu}\right)+\mathcal{O}\left(\Delta^{2}\right) \\
& \partial_{x}\left(h^{x y} \partial_{y} \bar{g}^{\mu \nu}\right)_{[I, J, K]}=D_{-x}\left(h_{[I+1 / 2, J, K]}^{x x} A_{+x} D_{0 y} \bar{g}_{[I, J, K]}^{\mu \nu}\right)+\mathcal{O}\left(\Delta^{2}\right) \\
& \partial_{x}\left(\frac{g^{x t}}{g^{t t}} Q^{\mu \nu}\right)_{[I, J, K]}=D_{-x}\left(\frac{g_{[I+1 / 2, J, K]}^{x t}}{g_{[I+1 / 2, J, K]}^{t t}} A_{+x} Q_{[I, J, K]}^{\mu \nu}\right)+\mathcal{O}\left(\Delta^{2}\right),
\end{aligned}
$$

where the averaging operator $A_{+x}$ is defined in appendix C.1. The code is evolved as a first differential order in time and second order in space system with a two-step iterated Crank-Nicholson algorithm or fourth-order Runge-Kutta integrator.

\section{B.3. HarmNaive}

The HarmNaive code is based upon harmonic coordinates but differs from the Abigel harm code because the evolution system consists of only the six wave equations (B.10) for the spatial components $\bar{g}^{i j}$. The time components are propagated by the harmonic conditions (B.8), i.e.,

$$
\partial_{t} \bar{g}^{\alpha t}+\partial_{i} \bar{g}^{\alpha i}=\hat{H}^{\alpha} \text {. }
$$

The coupling between $\bar{g}^{i j}$ and $\bar{g}^{\alpha t}$ makes the system only weakly hyperbolic.

The evolution equations for $\bar{g}^{i j}$ and $Q^{i j}$ are finite differenced as in the Abigel_harm code. The evolution equation (B.21) for $\bar{g}^{\alpha t}$ is approximated by central differences. The update scheme is a two-step iterative Crank-Nicholson algorithm.

\section{B.4. KrancNOR code}

B.4.1. Continuum formulation. Nagy, Ortiz and Reula suggested [17] modifications to the ADM system such that it can be made strongly hyperbolic whilst remaining in second-order form. The system we use includes slight adjustments of [9]. Additionally, we use an evolved lapse.

The variable $f_{i}$ is defined as

$$
f_{i}=\gamma^{k l}\left(\gamma_{i k, l}-\frac{1}{2} \rho \gamma_{k l, i}\right)
$$


with parameter $\rho$. This introduces the new constraint $G_{i}$ where

$$
G_{i}:=f_{i}-\gamma^{k l}\left(\gamma_{i k, l}-\frac{1}{2} \rho \gamma_{k l, i}\right) \text {. }
$$

Starting from the ADM evolution equations, an evolution equation for $f_{i}$ is obtained by differentiating (B.22) and commuting space and time derivatives. The Hamiltonian and momentum constraints are added with parameters $c$ and $b$, and derivatives of the $G_{i}$ are added with parameters $a$ and $a^{\prime}$,

$\partial_{t} \gamma_{i j}=-2 \alpha K_{i j}$

$\partial_{t} K_{i j}=-D_{i} D_{j} \alpha+\alpha\left(R_{i j}^{(3)}-2 K_{i k} K^{k}{ }_{j}+K_{i j} K\right)+\frac{a}{2} G_{(i, j)}+\left(c \mathcal{H}+a^{\prime} G_{k, l} \gamma^{k l}\right) \gamma_{i j}$

$\partial_{t} f_{i}=\alpha K^{k l}\left(2 \gamma_{i k, l}-\rho \gamma_{k l, i}\right)-\gamma^{k l}\left[2\left(\alpha K_{i k}\right),{ }_{l}-\rho\left(\alpha K_{k l}\right),{ }_{i}\right]+2 b \mathcal{M}_{i}$

$\partial_{t} \alpha=-\alpha F\left(\alpha, K, x^{i}\right)$.

The variables $\gamma_{i j}, K_{i j}, f_{i}$ and $\alpha$ are evolved. Due to the symmetries of $\gamma_{i j}$ and $K_{i j}$, this leads to 16 evolved variables. We write the Ricci tensor entirely in terms of $\gamma_{i j} ; f_{i}$ is only used where it appears as part of $G_{i}$.

For those tests requiring harmonic slicing, the lapse source function is

$$
F\left(\alpha, K, x^{i}\right)=\alpha K
$$

and for the expanding Gowdy test,

$$
F\left(\alpha, K, x^{i}\right)=K_{33} / \alpha,
$$

which is compatible with the exact lapse in this case. We make the following choice of parameters:

$$
a=1, \quad b=1, \quad a^{\prime}=0, \quad \rho=2 / 3, \quad c=0 .
$$

Note that choosing parameters

$$
a=0, \quad b=0, \quad a^{\prime}=0, \quad \rho=0, \quad c=0
$$

leads to a standard ADM system. This is useful for testing the code.

B.4.2. Semi-discrete scheme. To form the semi-discrete approximation, discretization in space is performed according to the standard second-order accurate discretization C.1.

Finite differences are taken only of the evolved variables $\gamma_{i j}, K_{i j}, f_{i}$ and $\alpha$. This means that where derivatives of other quantities appear, they are explicitly written in terms of derivatives of the evolved variables (e.g. by using the Leibniz rule).

We do not add Kreiss-Oliger-type artificial dissipation, as it was not necessary for stability.

B.4.3. Time integration. Time integration is performed using the method of lines with the iterative Crank-Nicholson (ICN) method.

B.4.4. Output. For our state vector $v=\left(\gamma_{i j}, K_{i j}, f_{i}\right)^{T}$ we define the $L_{2}$ - and $D_{+}$-norms,

$$
\begin{aligned}
\|v\|_{L_{2}}^{2} & \equiv \sum_{\text {grid }}\left(\eta^{i k} \eta^{j l} \gamma_{i j} \gamma_{k l}+\eta^{i k} \eta^{j l} K_{i j} K_{k l}+\eta^{i j} f_{i} f_{j}\right) h^{3} \\
\|v\|_{D_{+}}^{2} & \equiv\|v\|_{L_{2}}^{2}+\sum_{\text {grid }}\left(\eta^{i k} \eta^{j l} \eta^{m n} D_{+m} \gamma_{i j} D_{+n} \gamma_{k l}\right) h^{3}
\end{aligned}
$$


where $\eta \equiv \operatorname{diag}(1,1,1)$. This is the norm obtained from a reduction to first order [11] of the semi-discrete equations. The exact solution is denoted $u_{j}^{n} \equiv u\left(t^{n}, x_{j}\right)$ and the error is defined as

$$
\mathcal{E} \equiv v-u
$$

For the stability test, the exact solution is taken to be Minkowski in Cartesian coordinates. For those tests which are perturbations of this solution, we analyze relative error with respect to this background. We denote the background solution as $u_{B}$. Hence the relative error about this background is

$$
r \equiv \frac{\|\mathcal{E}\|_{L_{2}}}{\left\|u-u_{B}\right\|_{L_{2}}} .
$$

In general, we run until this quantity exceeds 0.2 (a relative error of $20 \%$ ).

\section{B.5. Family of BSSN (Shibata-Nakamura and Baumgarte-Shapiro) formulations}

The family of BSSN systems is constituted by variations of an evolution system that had originally been proposed by Nakamura in the late 1980s, and has been subsequently modified by Nakamura-Oohara and Shibata-Nakamura [44-46], and later by various other authors. The formulation is characterized by introducing a contracted connection term as a new variable, a conformal decomposition of the metric and extrinsic curvature variables, and adding constraints to the evolution equations. In particular, the system can be viewed as the NOR-system plus a conformal decomposition which leads to the evolution of a unimodular metric. The advantage of this formulation was re-announced by Baumgarte and Shapiro [47].

Modifications of the system have been obtained by variations in how derivatives of the new variables are written, how the gauge is specified, how algebraic constraints are treated, and the way (differential or algebraic) constraints are added to the evolution equations. A detailed discussion of well-posedness for the BSSN family has been given by Gundlach and Martin-Garcia [8-10], to which we refer for details about the BSSN family.

The set of evolved variables are the logarithm of the conformal factor $\varphi$, the conformally rescaled 3-metric $\tilde{\gamma}_{i j}$, the trace of the extrinsic curvature $K$, the conformally rescaled traceless extrinsic curvature $\tilde{A}_{i j}$ and the contracted Christoffel symbols $\tilde{\Gamma}^{i}$,

$$
\begin{aligned}
& \varphi=(1 / 12) \log \left(\operatorname{det} \gamma_{i j}\right), \\
& \tilde{\gamma}_{i j}=\mathrm{e}^{-4 \varphi} \gamma_{i j}, \\
& K=\gamma^{i j} K_{i j}, \\
& \tilde{A}_{i j}=\mathrm{e}^{-4 \varphi}\left(K_{i j}-(1 / 3) \gamma_{i j} K\right), \\
& \tilde{\Gamma}^{i}=\tilde{\Gamma}_{j k}^{i} \tilde{\gamma}^{j k}
\end{aligned}
$$

This immediately leads to the two algebraic constraints

$$
\operatorname{det} \gamma_{i j}=1, \quad \tilde{A}_{i}^{i}=0
$$

and the differential constraint

$$
\tilde{\Gamma}^{i}-\tilde{\gamma}^{j k} \tilde{\Gamma}_{j k}^{i}=0
$$

which are propagated by the evolution equations. Note that densitized quantities (those with a tilde) have their indices raised and lowered with the conformally rescaled 3-metric $\tilde{\gamma}_{i j}$.

The standard Hamiltonian and momentum constraints (B.3), (B.4) and (B.4) can be expressed in the BSSN variables as 


$$
\begin{aligned}
& \mathcal{H}=\mathrm{e}^{-4 \varphi} \tilde{R}-8 \mathrm{e}^{-4 \varphi} \tilde{D}^{j} \tilde{D}_{j} \varphi-8 \mathrm{e}^{-4 \varphi}\left(\tilde{D}^{j} \varphi\right)\left(\tilde{D}_{j} \varphi\right)+(2 / 3) K^{2}-\tilde{A}_{i j} \tilde{A}^{i j}-(2 / 3) \mathcal{A} K \\
& \mathcal{M}_{i}=6 \tilde{A}^{j}{ }_{i}\left(\tilde{D}_{j} \varphi\right)-2 \mathcal{A}\left(\tilde{D}_{i} \varphi\right)-(2 / 3)\left(\tilde{D}_{i} K\right)+\tilde{\gamma}^{k j}\left(\tilde{D}_{j} \tilde{A}_{k i}\right)
\end{aligned}
$$

The BSSN evolution equations, which are obtained from the ADM equations (B.1)-(B.4) by using definitions (B.32)-(B.36) and making a standard choice for adding constraints, are

$$
\begin{aligned}
& \mathcal{L}_{n} \varphi=-(1 / 6) \alpha K, \\
& \mathcal{L}_{n} \tilde{\gamma}_{i j}=-2 \alpha \tilde{A}_{i j}, \\
& \mathcal{L}_{n} K=-D^{i} D_{i} \alpha+\alpha \tilde{A}_{i j} \tilde{A}^{i j}+(1 / 3) \alpha K^{2}, \\
& \mathcal{L}_{n} \tilde{A}_{i j}=-\mathrm{e}^{-4 \varphi}\left(D_{i} D_{j} \alpha\right)^{T F}+\mathrm{e}^{-4 \varphi} \alpha\left(R_{i j}^{B S S N}\right)^{T F}+\alpha K \tilde{A}_{i j}-2 \alpha \tilde{A}_{i k} \tilde{A}^{k}{ }_{j}, \\
& \mathcal{L}_{n} \tilde{\Gamma}^{i}=-2\left(\partial_{j} \alpha\right) \tilde{A}^{i j}+2 \alpha\left(\tilde{\Gamma}_{j k}^{i} \tilde{A}^{k j}-(2 / 3) \tilde{\gamma}^{i j}\left(\partial_{j} K\right)+6 \tilde{A}^{i j}\left(\partial_{j} \varphi\right)\right),
\end{aligned}
$$

where $\tilde{D}_{i}$ is the covariant derivative associated with $\tilde{\gamma}_{i j}$, and $\mathcal{L}_{n}=\partial_{t}-\mathcal{L}_{\beta}$ is the Lie derivative along the unit normal. Note that $\int \mathcal{L}_{n} K d^{3} x$ is positive definite apart from boundary terms involving the lapse (which vanish for periodic boundary conditions). The Ricci curvature $R_{i j}^{B S S N}$ in terms of the BSSN variables becomes

$$
\begin{aligned}
& R_{i j}^{B S S N}=\tilde{R}_{i j}+R_{i j}^{\varphi}, \\
& R_{i j}^{\varphi}=-2 \tilde{D}_{i} \tilde{D}_{j} \varphi-2 \tilde{\gamma}_{i j} \tilde{D}^{k} \tilde{D}_{k} \varphi+4\left(\tilde{D}_{i} \varphi\right)\left(\tilde{D}_{j} \varphi\right)-4 \tilde{\gamma}_{i j}\left(\tilde{D}^{k} \varphi\right)\left(\tilde{D}_{k} \varphi\right), \\
& \tilde{R}_{i j}=-(1 / 2) \tilde{\gamma}^{l k} \partial_{l} \partial_{k} \tilde{\gamma}_{i j}+\tilde{\gamma}_{k(i} \partial_{j} \tilde{\Gamma}^{k}+\tilde{\Gamma}^{k} \tilde{\Gamma}_{(i j) k}+2 \tilde{\gamma}^{l m} \tilde{\Gamma}_{l(i}^{k} \tilde{\Gamma}_{j) k m}+\tilde{\gamma}^{l m} \tilde{\Gamma}_{i m}^{k} \tilde{\Gamma}_{k l j} .
\end{aligned}
$$

Note that there are different ways to numerically compute the trace free part of the Ricci tensor, e.g. one can project out the trace of the Ricci tensor according to

$$
R_{i j}^{T F}=R_{i j}-\frac{1}{3} R \gamma_{i j}
$$

compute the Ricci Scalar from the Hamiltonian constraint (B.39), or compute the trace free part explicitly by assuming the algebraic constraints hold.

We refer to the code descriptions below for details concerning the individual codes. In summary, the fundamental dynamical variables in $\operatorname{BSSN}$ are $\left(\varphi, \tilde{\gamma}_{i j}, K, \tilde{A}_{i j}, \tilde{\Gamma}^{i}\right)$, which total 17. The four gauge quantities are $\left(\alpha, \beta^{i}\right)$.

B.5.1. Concrete implementations. We have compared a number of codes based on variants of the BSSN system. Several of these are based on the Cactus computational toolkit [42]: the CCATIE_BSSN [48, 49] and Kranc_BSSN [50] codes, and the LazEv_BSSN [51] code. Of these, CCATIE_BSSN and Kranc_BSSN use the CactusMoL time integrator, which provides the RK3, RK4 and ICN methods, among others (see, e.g., [11]). Kranc_BSSN is based on the Kranc code generation software package [35].

All codes use straightforward replacement of partial derivatives by standard second-order centered finite differences with a three-point stencil (most codes are also able to use standard centered fourth-order finite differencing).

Most of the BSSN codes have a long history of use in production environments and have a large number of parameters that allow them great flexibility, e.g. regarding details of the numerical methods, gauge conditions, or the way the algebraic constraints are treated. Typical options to solve the algebraic constraints at every intermediate timestep use the following replacements: 
- Ensure that $\tilde{\gamma}_{i j}$ has unit determinant by setting

$$
\tilde{\gamma}_{i j} \rightarrow \frac{\tilde{\gamma}_{i j}}{\operatorname{det} \tilde{\gamma}^{1 / 3}}
$$

- Ensure that $\tilde{A}_{i j}$ remains trace free by setting

$$
\tilde{A}_{i j} \rightarrow \tilde{A}_{i j}-\frac{1}{3} \tilde{A}_{l m} \tilde{\gamma}^{i l} \tilde{\gamma}^{j m} .
$$

- Divide $\tilde{A}_{i j}$ by the same factor that is used to remove the determinant of $\tilde{\gamma}_{i j}$,

$$
\tilde{A}_{i j} \rightarrow \frac{\tilde{A}_{i j}}{\operatorname{det} \tilde{\gamma}^{1 / 3}} .
$$

Note that an ambiguity arises whenever $\Gamma^{i}$ or $\tilde{\gamma}^{k j} \gamma_{i j, k}$ occur, as they are related analytically by the equation $\Gamma^{i}=-\gamma^{i j},{ }_{j}-\frac{1}{2} \gamma^{i l}(\ln \gamma),{ }_{l}$. If the constraint $\gamma=1$ holds, e.g. if it is enforced at each timestep, this is equivalent numerically (up to round-off error) to $\Gamma^{i}=-\gamma^{i j},{ }_{j}$. Some authors replace $\gamma^{i j},{ }_{j}$ using $-\Gamma^{i}$ only when the expression appears under a derivative, but more complicated rules have also been applied.

Reference [52] describes a widely used combination of BSSN system and gauge condition in detail and examines this system's hyperbolicity.

\section{B.6. $\mathrm{KrancFN}$}

B.6.1. Continuum formulation. The Friedrich-Nagy system [53] is a frame-based first-order formulation that has been shown to yield a well-posed initial boundary value problem. The formulation starts from the four-dimensional vacuum equations

$$
\begin{aligned}
& T_{I J}^{\mu}:=\left[e_{I}, e_{J}\right]^{\mu}-\left(\Gamma_{I}{ }^{K}{ }_{J}-\Gamma_{J}{ }^{K}{ }_{I}\right) e_{K}{ }^{\mu}=0, \quad \mu=0,1,2,3 \\
& \Delta_{I J K L}:=R_{I J K L}(\Gamma)-C_{I J K L}=0 \\
& H_{J K L}:=\nabla_{I} C_{J K L}{ }^{I}=0, \quad I=0,1,2,3,
\end{aligned}
$$

where $e_{I}$ denote the tetrad vectors with coordinate components $e_{I}{ }^{\mu}$; and $\Gamma_{I}{ }_{J}{ }_{J}$ are the connection coefficients defined by $\nabla_{e_{I}} e_{K}=\Gamma_{I}{ }^{J}{ }_{K} e_{J}$ and satisfying $\eta_{J}{ }_{M} \Gamma_{I}{ }^{J}{ }_{K}+\eta_{K}{ }_{J} \Gamma_{I}{ }^{J}{ }_{M}=0$. $R_{I J K L}$ and $C_{I J K L}$ denote the components of the Riemann and Weyl tensor with respect to the tetrad. The Riemann tensor is given in terms of the connection coefficients by

$$
\begin{aligned}
R_{I J}{ }_{K}{ }_{K}(\Gamma)= & e_{I}\left(\Gamma_{J}{ }^{L}{ }_{K}\right)-e_{J}\left(\Gamma_{I}{ }^{L}{ }_{K}\right) \\
& -\Gamma_{M}{ }^{L}{ }_{K} \Gamma_{I}{ }^{M}{ }_{J}-\Gamma_{I}{ }^{M}{ }_{K} \Gamma_{J}{ }^{L}{ }_{M}+\Gamma_{M}{ }_{K}{ }_{K} \Gamma_{J}{ }^{M}{ }_{I}+\Gamma_{I}{ }^{L}{ }_{M} \Gamma_{J}{ }^{M}{ }_{K} .
\end{aligned}
$$

Equation (B.50) states that the connection is torsion free, (B.51) are the vacuum Einstein equations and (B.52) is the Bianchi identity for a vacuum spacetime. From (B.50)-(B.52), a symmetric hyperbolic evolution system is obtained by choosing certain combinations of components of the above equations as well as a gauge that is adapted to the boundary.

Assuming a boundary at $z=$ const, we foliate the interior domain by timelike hypersurfaces $T_{c}$ given by $z=c=$ const. The frame is adapted to this foliation and boundary such that the frame vector $e_{3}$ is orthogonal to $T_{c}$, which implies for the coordinate components

$$
e_{a}^{3}=0, \quad a=0,1,2, \quad e_{3}^{3}>0,
$$

with $e_{3}$ being the unit normal to $T_{c}$ implies $\left.\Gamma_{a}{ }^{3} b=\Gamma_{(a}{ }^{3} b\right)$. 
The mean extrinsic curvature of $T_{c}$ is prescribed as a function of the coordinates $f\left(x^{\mu}\right)$ and used to eliminate the connection coefficient $\Gamma_{0}{ }_{0}{ }_{0}$ from the equations

$$
\Gamma_{0}{ }^{3}=f+\Gamma_{1}{ }^{3}{ }_{1}+\Gamma_{2}{ }^{3} \text {. }
$$

The variation of $e_{0}$ within $T_{c}$ is prescribed by functions $F^{A}\left(x^{\mu}\right), A=1,2$ according to $D_{e_{0}} e_{0}=F^{A} e_{A}$, where $D$ denotes the induced connection on $T_{c}$. This eliminates the connection coefficients

$$
\Gamma_{0}^{A}{ }_{0}=F^{A}, \quad A=1,2 .
$$

The tetrad vectors $e_{A}$ are Fermi-transported along $e_{0}$ with respect to $D$ and therefore

$$
\Gamma_{0}{ }_{B}^{A}=0, \quad A, B=1,2 .
$$

The coordinates $\left\{x^{\mu}\right\}$ are chosen such that the tetrad vector $e_{0}$ represents the time flow $\partial_{t}$, i.e.,

$$
e_{0}{ }^{\mu}=\delta_{0}^{\mu} \text {. }
$$

The ten independent components of the Weyl tensor are encoded in the symmetric and tracefree tensor fields

$$
E_{i j}:=C_{i 0 j 0}, \quad B_{i j}:=\frac{1}{2} C_{0 i k l}{ }^{(3)} \epsilon_{j}^{k l}
$$

corresponding to the electric and magnetic parts with respect to $e_{0}$. The conditions $\delta^{i j} E_{i j}=\delta^{i j} B_{i j}=0$ are incorporated explicitly by eliminating

$$
E_{33}=-\left(E_{11}+E_{22}\right), \quad B_{33}=-\left(B_{11}+B_{22}\right)
$$

from the equations. In total the Friedrich-Nagy system has 37 variables, namely

$$
\left.\mathbf{u}=\left(e_{A}{ }^{p}, e_{3}{ }^{\mu}, \Gamma_{i}{ }^{0}{ }_{j}, \Gamma_{3}{ }^{i}{ }_{j}, \Gamma_{(A}{ }^{3} B\right), \Gamma_{A}{ }^{B}{ }_{C}, E_{i A}, B_{i A}\right)^{T},
$$

where

$$
A, B, C=1,2, \quad i, j=1,2,3, \quad p=0,1,2, \quad \mu=0,1,2,3 .
$$

A symmetric hyperbolic evolution system for the variables (B.60) is obtained by taking the following combinations of (B.50)-(B.52):

$$
\begin{aligned}
& T_{0 A}{ }^{p}=0, \quad T_{03}{ }^{\mu}=0, \quad \Delta_{0 B a b}=0, \quad \Delta_{0131}=0, \quad \Delta_{0232}=0, \\
& \Delta_{0132}+\Delta_{0231}=0, \quad \Delta_{0130}+\Delta_{1232}=0, \quad \Delta_{0230}+\Delta_{2131}=0, \\
& \Delta_{A B 03}=0, \quad \Delta_{A 003}=0, \quad \Delta_{3 A 03}+\Delta_{303 A}=0, \quad \eta^{a b} \Delta_{3 a b 3}=0, \\
& H_{0 i j}-\frac{1}{2} \delta^{3}{ }_{i} \epsilon_{j)}{ }^{3 l} H_{m n 0} \epsilon^{m n}{ }_{l}=0, \quad \frac{1}{2} H_{m k i} \epsilon^{m k}{ }_{j}+\delta^{3}{ }_{i} \epsilon_{j)}{ }^{3 m} H_{0 m 0}=0,
\end{aligned}
$$

where the convention for the indices is the same as in equation (B.60) and $a, b=0,1,2$. The resulting system is given explicitly in $[53,36]$ and is of the form

$$
\mathbf{A}^{0} \partial_{t} \mathbf{u}+\mathbf{A}^{i} \partial_{i} \mathbf{u}+\mathbf{B}(\mathbf{u}, F)=0,
$$

where $F=\left(f, F^{A}, \partial_{\mu} f, \partial_{\mu} F^{A}\right)$ represents the gauge source functions and their derivatives. The matrices $\mathbf{A}^{0}, \mathbf{A}^{i}$ are symmetric and depend on the coordinate components of the frame. $\mathbf{A}^{0}$ is positive definite as long as $1-\left(e_{1}\right)^{2}-\left(e_{2}\right)^{2}-\left(e_{3}\right)^{2}>0$, which corresponds to $e_{0}$ being timelike. Characteristics are timelike and null.

The remaining components of (B.50)-(B.52),

$$
T_{i j}{ }^{\mu}=0, \quad \Delta_{i j}{ }^{L}{ }_{K}=0, \quad H_{0 k 0}=0, \quad \frac{1}{2} H_{j k 0} \epsilon^{j k}{ }_{m}=0,
$$

only contain derivatives in directions orthogonal to $e_{0}$ and are satisfied if satisfied initially by virtue of the evolution equations (see [53]). $e_{0}$ in general is not hypersurface orthogonal and therefore the constraints do contain derivatives in direction of $\partial_{t}$. In order to monitor these constraints during a numerical evolution, we eliminate the time derivatives by means of the evolution equations. 
B.6.2. Numerical implementation. The code is based on the Cactus Computational Toolkit [42] and the Kranc software [35, 36]. The spatial discretization of (B.61) is done in a straightforward way

$$
\partial_{t} \mathbf{u}=-\left(\mathbf{A}^{0}\right)^{-1} \mathbf{A}^{i} D_{i} \mathbf{u}+\left(\mathbf{A}^{0}\right)^{-1} \mathbf{B}(\mathbf{u}, F),
$$

where $D_{i}$ is the second- (or fourth-order) accurate centered derivative operator in the direction $i$ (see appendix C.1). Time integration is done with the method of lines (CactusMoL) using ICN for the second-order scheme and RK4 for the fourth-order scheme. If needed, artificial dissipation is added to the right-hand side of equation (B.62) in the form

$$
\left(\mathbf{A}^{0}\right)^{-1} Q_{d} \mathbf{u}
$$

where $Q_{d}$ is the Kreiss-Oliger dissipation operator (see appendix C.2). Respecting the symmetrizer in the dissipation term is essential; replacing it by the identity matrix triggered exponentially growing continuum modes, e.g. for the gauge wave testbed with nonlinear amplitude.

\section{B.7. LSU_HyperGR}

This symmetric hyperbolic first-order formulation is described by Sarbach and Tiglio in [54]. The system has 34 evolved variables which are the standard ADM metric $\gamma_{i j}$, extrinsic curvature $K_{i j}$ and lapse $\alpha$, as well as extra variables $d_{k i j}=\partial_{k} \gamma_{i j}$ and $A_{i}=\partial_{i} \alpha / \alpha$, introduced to make the formulation first order in space.

In addition to the Hamiltonian constraint $\mathcal{H}$ and the momentum constraint $\mathcal{M}_{i}$, the constraints arising from those new variables are

$$
\begin{aligned}
& C_{A_{i}}=A_{i}-\partial_{i} \alpha / \alpha, \\
& C_{k i j}=d_{k i j}-\partial_{k} \gamma_{i j}, \\
& C_{l k i j}=\partial_{[l} d_{k] j k} .
\end{aligned}
$$

The system of PDEs resulting from the standard ADM $(3+1)$-decomposition of the Einstein equations is only weakly hyperbolic. To get a symmetric hyperbolic system the principal part has to be modified further. This is done by adding the constraints to the righthand sides of the evolution equations with appropriate multiplicative factors $\zeta, \xi, \eta, \chi$ and $\iota$. Here these parameters are chosen to be constant in space, although in general this is not necessary. The full set of equations is then

$\partial_{0} \gamma_{i j}=-2 K_{i j}$

$\partial_{0} K_{i j}=R_{i j}-\frac{1}{\alpha} \nabla_{i} \nabla_{j} \alpha-2 K_{i a} K_{j}^{a}+K K_{i j}+\iota \gamma_{i j} \mathcal{H}+\zeta \gamma^{a b} C_{a(i j) b}$,

$\partial_{0} d_{k i j}=-2 \partial_{k} K_{i j}-2 A_{k} K_{i j}+\eta \gamma_{k(i} \mathcal{M}_{j)}+\chi \gamma_{i j} \mathcal{M}_{k}$,

$\partial_{0} \alpha=-F\left(\alpha, K, x^{\mu}\right)+S\left(x^{\mu}\right)$,

$\partial_{0} A_{i}=-\frac{\partial F\left(\alpha, K, x^{\mu}\right)}{\partial \alpha} A_{i}-\frac{1}{\alpha} \frac{\partial F\left(\alpha, K, x^{\mu}\right)}{\partial K} \partial_{i} K-\frac{1}{\alpha} \frac{\partial F\left(\alpha, K, x^{\mu}\right)}{\partial x^{i}}+\xi \mathcal{M}_{i}$,

where $\partial_{0}=\left(\partial_{t}-\mathcal{L}_{\beta}\right) / \alpha, R_{i j}$ is the Ricci tensor and $K$ is the trace of the extrinsic curvature. The functions $F\left(\alpha, K, x^{i}\right)$ and $S\left(x^{i}\right)$ are pure gauge and can be chosen freely. The choices $S=0$ and $F=\alpha K$ provides harmonic gauge conditions. 
Restriction of the parameters $\chi, \xi, \eta, \zeta, \iota$ to the family

$$
\iota=-1 / 2, \quad \zeta \eta=-2, \quad \xi=-1 / 2 \chi+1 / 4 \eta-1 / 2
$$

results in a strongly hyperbolic system. A symmetric hyperbolic subfamily is given by $\zeta=-1$, which leaves $\chi$ as the single free parameter (constrained only by the condition $\chi \neq 0$ ). The runs presented here were done with the specific choice of $\chi=-1$.

To ensure a numerically stable discretization based on the energy method for hyperbolic equations, second-order spatial differencing operators that satisfy the summation by parts (SBP) condition are used [55, 56].

Furthermore, a small amount of dissipation (standard Kreiss-Oliger dissipation operators) is added to the right-hand sides of the evolution equations.

The integration in time is done with a third-order Runge-Kutta scheme.

\section{Appendix C. Numerical methods}

\section{C.1. Spatial discretization}

Most of our numerical results are based on second-order accurate centered discretization,

$$
\partial_{i} \rightarrow D_{0 i}, \quad \partial_{i} \partial_{j} \rightarrow \begin{cases}D_{0 i} D_{0 j} & \text { if } \quad i \neq j \\ D_{+i} D_{-i} & \text { if } \quad i=j,\end{cases}
$$

where

$$
\begin{aligned}
& D_{+} v_{j}:=\frac{v_{j+1}-v_{j}}{\Delta x}, \\
& D_{-} v_{j}:=\frac{v_{j}-v_{j-1}}{\Delta x}, \\
& D_{0} v_{j}:=\frac{v_{j+1}-v_{j-1}}{2 \Delta x}, \\
& D_{+} D_{-} v_{j}:=\frac{v_{j+1}-2 v_{j}+v_{j-1}}{\Delta x^{2}} .
\end{aligned}
$$

For a summary of definitions and results for standard fourth-order discretizations we again refer to [11], where in particular some results concerning the evolution systems considered here are derived.

Finally, averaging operators $A_{ \pm}$are defined as

$$
\begin{aligned}
& A_{+} v_{j}:=\frac{v_{j+1}+v_{j}}{2} \\
& A_{-} v_{j}:=\frac{v_{j}+v_{j-1}}{2} .
\end{aligned}
$$

\section{C.2. Artificial dissipation}

For second-order accurate codes, it is common practice to add third-order accurate KreissOliger dissipation [57] to all right-hand sides of the time evolution equations as

$$
\partial_{t} \mathbf{u} \rightarrow \partial_{t} \mathbf{u}+Q \mathbf{u}
$$


Here we use the following general form of the Kreiss-Oliger dissipation operator $Q$ of order $2 r$ :

$$
Q=\sigma(-1)^{r} h^{2 r-1}\left(D_{+}\right)^{r} \rho\left(D_{-}\right)^{r} / 2^{2 r},
$$

for a $2 r-2$ accurate scheme, where the parameter $\sigma$ regulates the strength of the dissipation and $\rho$ is a weighting function, which is typically set to 1 in the interior but may go to 0 at a boundary. Since we mostly focus on second-order accurate codes here, the relevant case is $r=2$, for which

$$
Q=-\sigma h^{3}\left(D_{+}\right)^{2} \rho\left(D_{-}\right)^{2} / 16,
$$

which may be implemented using Erik Schnetter's Cactus thorn AEIThorns/Dissipation [38].

\section{References}

[1] Hahn S G and Lindquist R W 1964 The two body problem in geometrodynamics Ann. Phys. 29 304-31

[2] Pretorius F 2005 Evolution of binary black hole spacetimes Phys. Rev. Lett. 95121101

[3] Campanelli M, Lousto C O, Marronetti P and Zlochower Y 2006 Accurate evolutions of orbiting black-hole binaries without excision Phys. Rev. Lett. 96111101

[4] Baker J G, Centrella J, Choi D-I, Koppitz M and van Meter J 2006 Gravitational wave extraction from an inspiraling configuration of merging black holes Phys. Rev. Lett. 96111102

[5] Apples With Apples: Numerical Relativity Comparisons and Tests, http://www.ApplesWithApples.org

[6] Alcubierre M et al 2004 Towards standard testbeds for numerical relativity Class. Quantum Grav. 21 589-613

[7] Beyer H and Sarbach O 2004 On the well posedness of the Baumgarte-Shapiro-Shibata-Nakamura formulation of Einstein's field equations Phys. Rev. D 70104004

[8] Gundlach C and Martin-Garcia J M 2004 Symmetric hyperbolic form of systems of second-order evolution equations subject to constraints Phys. Rev. D 70044031

[9] Gundlach C and Martin-Garcia J M 2004 Symmetric hyperbolicity and consistent boundary conditions for second-order Einstein equations Phys. Rev. D 70044032

[10] Gundlach C and Martin-Garcia J M 2006 Hyperbolicity of second-order in space systems of evolution equations Class. Quantum Grav. 23 S387-404

[11] Calabrese G, Hinder I and Husa S 2006 Numerical stability for finite difference approximations of Einstein's equations J. Comp. Phys. 218 607-34

[12] Szilágyi B, Kreiss H-O and Winicour J 2005 Modeling the black hole excision problem Phys. Rev. D 71104035

[13] Motamed M, Babiuc M C, Szilagyi B, Kreiss H-O and Winicour J 2006 Finite difference schemes for second order systems describing black holes Phys. Rev. D 73124008

[14] Computational Fluid Dynamics Wiki/Validation and test cases CFD Online http://www.cfd-online.com/Wiki/ Validation_and_test_cases

[15] Test set for IVP solvers. http://pitagora.dm.uniba.it/ testset/

[16] Courant R and Friedrichs K O 1976 Supersonic Flows and Shock Waves (Berlin: Springer)

[17] Nagy G, Ortiz O E and Reula O A 2004 Strongly hyperbolic second order Einstein's evolution equations Phys. Rev. D 70044012

[18] Gundlach C and Martin-Garcia J M 2006 Well-posedness of formulations of the Einstein equations with dynamical lapse and shift conditions Phys. Rev. D 74024016

[19] Calabrese G, Pullin J, Sarbach O and Tiglio M 2002 Convergence and stability in numerical relativity Phys. Rev. D 66041501

[20] Gustafsson B, Kreiss H-O and Oliger J 1995 Time Dependent Problems and Difference Methods (New York: Wiley)

[21] Babiuc M C, Szilágyi B and Winicour J 2006 Some mathematical problems in numerical relativity Lect. Notes Phys. 692 251-74

[22] Husa S, Schneemann C, Vogel T and Zenginoglu A 2005 Hyperboloidal data and evolution Proc. 2005 Spanish Relativity Meeting, AIP Conf. Proc. 841306

[23] Babiuc M C, Szilágyi B and Winicour J 2006 Testing numerical relativity with the shifted gauge wave Class. Quantum Grav. 23 S319-42

[24] Babiuc M C, Szilágyi B and Winicour J 2006 Harmonic initial-boundary evolution in general relativity Phys. Rev. D 73064017 
[25] Boyle M, Lindblom L, Pfeiffer H, Scheel M and Kidder L 2007 Testing the accuracy and stability of spectral methods in numerical relativity Phys. Rev. D 75 024006-18

[26] Kidder L E, Scheel M A and Teukolsky S A 2001 Extending the lifetime of 3D black hole computations with a new hyperbolic system of evolution equations Phys. Rev. D 64064017

[27] Gowdy R H 1971 Phys. Rev. Lett. 27826

[28] Ringstrom H 2004 On a wave map equation arising in general relativity Commun. Pure Appl. Math. 57657

[29] Berger B K 2002 Asymptotic behavior of a class of expanding gowdy spacetimes Preprint gr-qc/0207035v1

[30] Frauendiener J and Vogel T 2005 Algebraic stability analysis of constraint propagation Class. Quantum Grav. 22 1769-93

[31] Husa S 2002 Current Trends in Relativistic Astrophysics (Lecture Notes in Physics vol 617) ed L Fernández and L Manuel González (Berlin: Springer)

[32] Garfinkle D 2002 Harmonic coordinate method for simulating generic singularities Phys. Rev. D 65044029

[33] Post D E and Votta L G 2005 Computational science demands a new paradigm Phys. Today. 5835

[34] Rinne O 2006 Stable radiation-controlling boundary conditions for the generalized harmonic Einstein equations Class. Quantum Grav. 23 6275-300

[35] Husa S, Hinder I and Lechner C 2006 Kranc: a mathematica application to generate numerical codes for tensorial evolution equations Comput. Phys. Comm. 174 983-1004

[36] Alic D 2005 Toward the numerical implementation of well-posed, constraint preserving evolution systems for general relativity Master's thesis University of Timisoara

[37] Hinder I 2005 Well-posed formulations and stable finite differencing schemes for numerical relativity PhD thesis University of Southampton, Southampton, UK

[38] Schnetter E AEIThorns/Dissipation Cactus thorn

[39] Arnowitt R, Deser S and Misner C W 1962 The dynamics of general relativity Gravitation: An Introduction to Current Research ed L Witten (New York: Wiley) pp 227-65

[40] York J W 1979 Kinematics and dynamics of general relativity Sources of Gravitational Radiation ed L L Smarr (Cambridge: Cambridge University Press) pp 83-126

[41] Frittelli S 1997 Note on the propagation of the constraints in standard $3+1$ general relativity Phys. Rev. D 55 5992-6

[42] Cactus Computational Toolkit http://www.cactuscode.org

[43] Szilágyi B and Winicour J 2003 Well-posed initial-boundary evolution in general relativity Phys. Rev. D 68041501

[44] Nakamura T, Oohara K-i and Kojima Y 1987 General relativistic collapse to black holes and gravitational waves from black holes Prog. Theor. Phys. Suppl. 90 1-218

[45] Nakamura T and Oohara K-i 1989 Methods in 3D numerical relativity Frontiers in Numerical Relativity ed C Evans, L Finn and D Hobill (Cambridge: Cambridge University Press) pp 254-80

[46] Shibata M and Nakamura T 1995 Evolution of three-dimensional gravitational waves: harmonic slicing case Phys. Rev. D 525428

[47] Baumgarte T W and Shapiro S L 1999 On the numerical integration of Einstein's field equations Phys. Rev. D 59024007

[48] Alcubierre M, Brügmann B, Dramlitsch T, Font J A, Papadopoulos P, Seidel E, Stergioulas N and Takahashi R 2000 Towards a stable numerical evolution of strongly gravitating systems in general relativity: the conformal treatments Phys. Rev. D 62044034

[49] Alcubierre M, Brügmann B, Diener P, Koppitz M, Pollney D, Seidel E and Takahashi R 2003 Gauge conditions for long-term numerical black hole evolutions without excision Phys. Rev. D 67084023

[50] Husa $S$ The FlexBSSN code is available on request from the author

[51] Zlochower Y, Baker J G, Campanelli M and Lousto C O 2005 Accurate black hole evolutions by fourth-order numerical relativity Phys. Rev. D 72024021

[52] Brown D, Sarbach O, Schnetter E, Tiglio M, Diener P, Hawke I and Pollney D 2007 Excision without excision Phys. Rev. D 76081503

[53] Friedrich H and Nagy G 1999 The initial boundary value problem for Einstein's vacuum field equations Commun. Math. Phys. 201 619-55

[54] Sarbach O and Tiglio M 2002 Exploiting gauge and constraint freedom in hyperbolic formulations of Einstein's equations Phys. Rev. D 66064023

[55] Strand B 1994 Summation by parts for finite differencing approximations for d/dx J. Comput. Phys. 11047

[56] Lehner L, Reula O and Tiglio M 2005 Multi-block simulations in general relativity: high order discretizations, numerical stability, and applications Class. Quantum Grav. 22 5283-322

[57] Kreiss H O and Oliger J 1973 Methods for the approximate solution of time dependent problems GARP publication series no 10 , Geneva 